\title{
FMO rewires metabolism to promote longevity through tryptophan and one carbon metabolism
}

\section{Authors:}

Hyo Sub Choi ${ }^{1 *}$, Ajay Bhat ${ }^{1 *}$, Marshall B. Howington ${ }^{2 *}$, Megan L. Schaller ${ }^{1}$, Rebecca Cox ${ }^{1}$, Shijiao Huang ${ }^{1}$, Safa Beydoun ${ }^{1}$, Hillary A. Miller ${ }^{2}$, Angela M. Tuckowski ${ }^{2}$, Joy Mecano ${ }^{1}$, Elizabeth S. Dean ${ }^{1}$, Lindy Jensen ${ }^{1}$, Daniel A. Beard ${ }^{1}$, Charles R. Evans ${ }^{3}$, Scott F. Leiser ${ }^{1,3}$

* Denotes equal contributions

\section{Affiliations:}

1. Department of Molecular and Integrative Physiology, University of Michigan, Ann Arbor, Ml 48109, USA.

2. Cellular and Molecular Biology Program, University of Michigan, Ann Arbor, MI 48109, USA.

3. Department of Internal Medicine, University of Michigan, Ann Arbor, MI 48109, USA.

\section{Keywords:}

aging, metabolism, flavin containing monooxygenase, one carbon metabolism, tryptophan, kynurenine, stress resistance, lifespan 


\section{Abstract}

28 Flavin containing monooxygenases (FMOs) are promiscuous enzymes known for metabolizing

29 a wide range of exogenous compounds. In C. elegans, fmo-2 expression increases lifespan and

30 healthspan downstream of multiple longevity-promoting pathways through an unknown

31 mechanism. Here, we report that, contrary to its classification as a xenobiotic enzyme, fmo-2

32 expression leads to rewiring of endogenous metabolism principally through changes in one

33 carbon metabolism (OCM). Using computer modeling, we identify decreased methylation as the

34 major OCM flux modified by FMO-2 that is sufficient to recapitulate its longevity benefits. We

35 further find that tryptophan is decreased in multiple mammalian FMO overexpression models

36 and is a validated substrate for FMO enzymes. Our resulting model connects a single enzyme

37 to two previously unconnected key metabolic pathways and provides a framework for the

38 metabolic interconnectivity of longevity-promoting pathways such as dietary restriction. FMOs

39 are well-conserved enzymes that are also induced by lifespan-extending interventions in mice,

40 supporting a conserved and critical role in promoting health and longevity through metabolic

41 remodeling. 
Flavin-containing monooxygenases (FMOs) are a family of enzymes that oxygenate substrates with nucleophilic centers, such as nitrogen and sulfur ${ }^{1}$. They were first discovered 50 years ago and have been studied extensively under the context of xenobiotic and drug

47 metabolism ${ }^{1}$. FMOs bind to an FAD prosthetic group and interact with an NADPH cofactor to

48 oxygenate substrates ${ }^{2}$. The FMO protein family is highly conserved both genetically and

49 structurally from bacteria to humans ${ }^{2,3}$. Considering the conserved nature of FMOs, it is

50 plausible that they share an endogenous, more ancient physiological role than detoxifying

51 xenobiotics.

52 Through a screen of genes downstream the hypoxia-inducible factor-1 (HIF-1), a

53 longevity-promoting transcription factor in C. elegans, flavin-containing monooxygenase-2 (fmo-

54 2) was identified as necessary for the longevity and health benefits of both hypoxia and dietary

55 restriction $(\mathrm{DR})^{4}$. The fmo-2 gene is also sufficient to confer these benefits on its own when

56 overexpressed ${ }^{4}$. Recently, studies also suggest potential endogenous role(s) for mammalian

57 FMOs, where changes in expression of multiple FMO proteins affect systemic metabolism ${ }^{5-10}$.

58 Initial correlative reports also link FMOs to the aging process, showing that Fmo genes are

59 frequently induced in long-lived mouse models, such as DR mice ${ }^{5,6}$. However, the

60 mechanism(s) for how Fmos modulate endogenous metabolism and/or aging in vivo is unclear,

61 as is their potential to benefit health and longevity in multiple species. While frequently implicated in cancer cells, recent studies identify one carbon

63 metabolism (OCM) as a common downstream target of multiple longevity pathways ${ }^{11-14}$. OCM is

64 an important intermediate metabolic pathway and refers to a two-cycle metabolic network

65 including the folate cycle and the methionine cycle ${ }^{15}$. OCM takes nutrient inputs, including

66 glucose and vitamin B12, and utilizes them to synthesize intermediates for metabolic processes

67 involved in growth and survival, including nucleotide metabolism, the transsulfuration and

68 transmethylation pathways, and lipid metabolism ${ }^{12,13,16}$. In particular, suppressing expression of 
69 the methionine cycle gene sams-1 by RNA-mediated interference (RNAi) extends the wild type 70 worm lifespan, but fails to further extend the lifespan of the genetic DR model eat-2 mutants ${ }^{17}$.

71 Kynurenine synthesis from tryptophan and subsequent metabolism is another important

72 metabolic pathway that can play a role in many processes, including longevity regulation.

73 Knocking out tryptophan 2,3-dioxygenase (TDO), which catalyzes the first and rate-limiting step

74 of this pathway, leads to lifespan extension in worms and flies ${ }^{18,19}$. Similarly, suppressing the

75 kynurenine pathway by knocking down kynureninase (kynu-1) in worms also increases

76 lifespan ${ }^{20}$. The kynurenine pathway competes for tryptophan with the serotonergic biosynthesis

77 pathway and produces nicotinamide adenine dinucleotide (NAD) and other metabolites,

78 including kynurenic acid and picolinic acid ${ }^{21}$.

Given that 1) induction of Fmos correlates with increased longevity across species, 2)

80 nematode fmo-2 is necessary and sufficient to improve health and longevity downstream of

81 metabolic perturbations, and 3) loss of Fmo expression can modify aspects of metabolism, we

82 hypothesized that Fmos affect aging by modifying one or more distinct metabolic processes.

83 Therefore, we sought to determine the metabolic changes that occur when the expression of

84 nematode fmo-2 is perturbed to identify its mechanism of longevity regulation. Our resulting

85 data support a model where fmo-2 oxygenates tryptophan, leading to alteration of OCM

86 components that confer longevity and healthspan benefits by reducing flux through methylation

87 processes. 


\section{$\underline{\text { Results }}$}

\section{Fmo-2 alters one carbon metabolism}

Based on the conserved enzymatic mechanism ${ }^{2,3}$ and our published data supporting a key role for nematode FMO-2 in regulating stress resistance, healthspan and longevity ${ }^{4}$, we hypothesized that FMO-2 may significantly alter endogenous metabolism in C. elegans. To test if systemic metabolism was broadly altered by FMO-2, we used untargeted metabolomics analysis (Supplementary Data 1) of three strains with varying fmo-2 expression: the wild type reference strain (N2 Bristol) the fmo-2(ok2147) putative knockout strain (FMO-2 KO), and our previously published long-lived fmo-2 overexpression (KAE9) strain (FMO-2 OE). The resulting principal component analysis (PCA) shows a substantial explained variance $(65.3 \%)$ through principal components (PC) 1 and 2 (Figure 1A). Our untargeted metabolomics data suggest a distinct difference in the metabolome between the three strains, consistent with expression of nematode fmo-2 being sufficient to modify endogenous metabolism (Figure 1B). what key metabolic aspects are modified. Using p-value $<0.05$ as our significance threshold, we identified five metabolic pathways that are significantly altered by the overexpression of fmo2, most of which are involved in amino acid metabolism (Figure 1C, Supplementary Data 2).

106 Of the five pathways, we observed the most significant enrichment in glycine, serine, and 107 threonine metabolism (Figure 1C). Exogenous supplementation of glycine in worm diet is 108 reported to extend lifespan by remodeling the methionine cycle ${ }^{22}$, a component of one carbon 109 metabolism (OCM) and another significantly enriched metabolic pathway from our analysis, 110 cysteine and methionine metabolism (Figure 1C, Supplementary Data 2). Indeed, OCM is a 111 nexus of multiple metabolic pathways that are necessary for survival; OCM is implicated in 112 multiple longevity pathways, including dietary restriction, insulin/IGF-1 signaling, and the 113 metformin-induced longevity response ${ }^{13,16,23}$. Due to its relevance in multiple longevity pathways 
114 and the direct involvement of cysteine and methionine metabolism within this metabolic network,

115 we postulated that fmo-2 regulates longevity through its interactions with OCM.

116 To test whether fmo-2 expression modifies OCM, we used targeted metabolomics

117 analysis on a panel of metabolites involved in OCM and related pathways to determine whether

118 their abundance levels were altered following fmo-2 expression (Supplementary Data 3). We

119 hypothesized that the affected metabolites would have abundance levels that correlate with

120 fmo-2 expression level. Thus, we compared the level of metabolite abundance between the wild

121 type and FMO-2 OE and also between the wild type and FMO-2 KO. Consistent with our

122 hypothesis that OCM is altered by fmo-2 expression, we observed significant changes in the

123 abundance level of cystine, homocysteine, s-adenosylmethionine (SAM), and thiamine in FMO-

1242 OE compared to the wild type (Figure 1D). With the exception of cystine, we observed

125 insignificant but consistent trends in the abundance level of these metabolites that moved in the

126 opposite direction in FMO-2 KO compared to FMO-2 OE (Figure 1D). The insignificant trends in

127 FMO-2 KO are consistent with our previous observation that knocking out fmo-2 does not affect

128 worm lifespan ${ }^{4}$. Similarly, we also observed insignificant but consistent trends in the abudance

129 level that correlate with fmo-2 expression level in multiple other metabolites that we measured

130 (Supplementary Figure 1). Taken together, our results are consistent with the hypothesis that

131 the OCM pathway is modified by fmo-2 expression. 
A

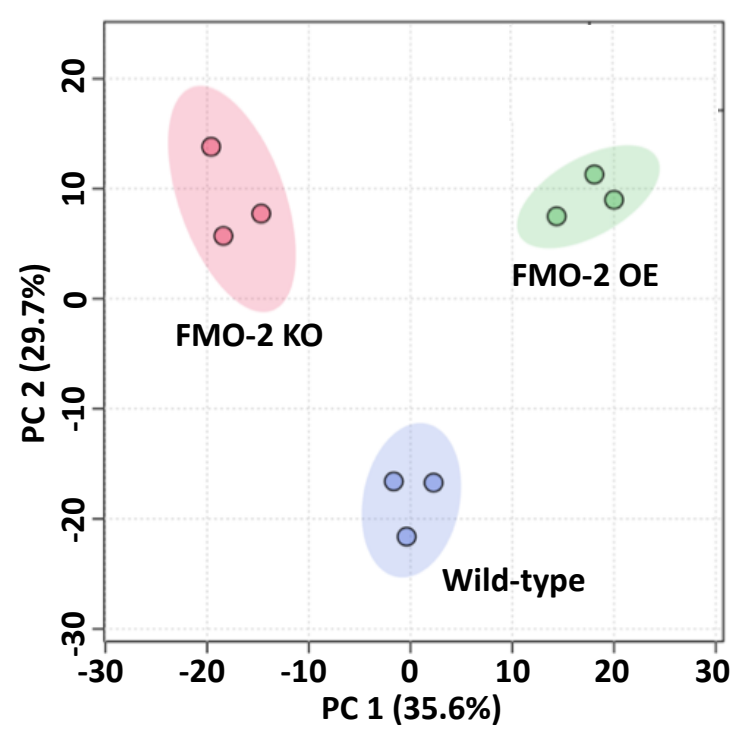

C

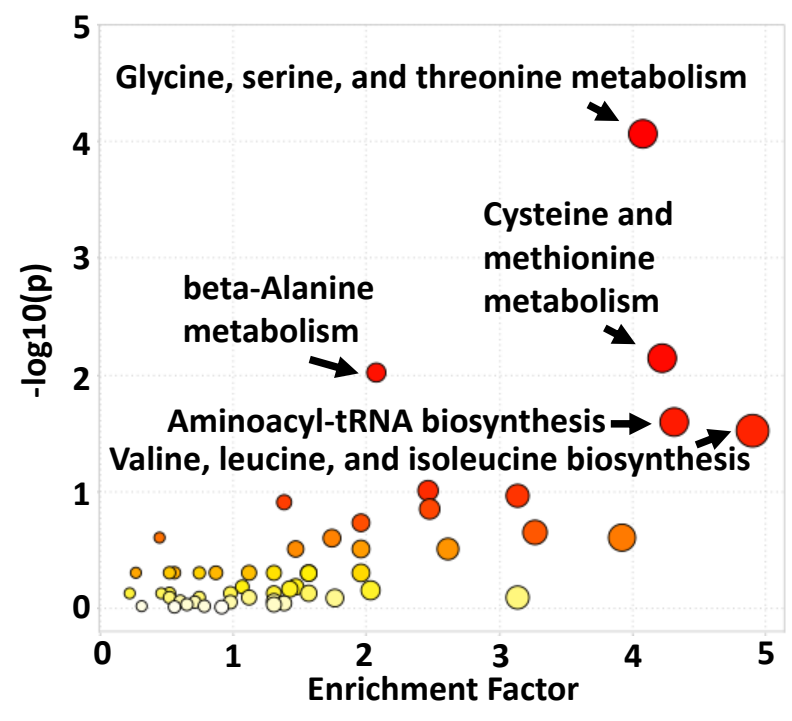

B

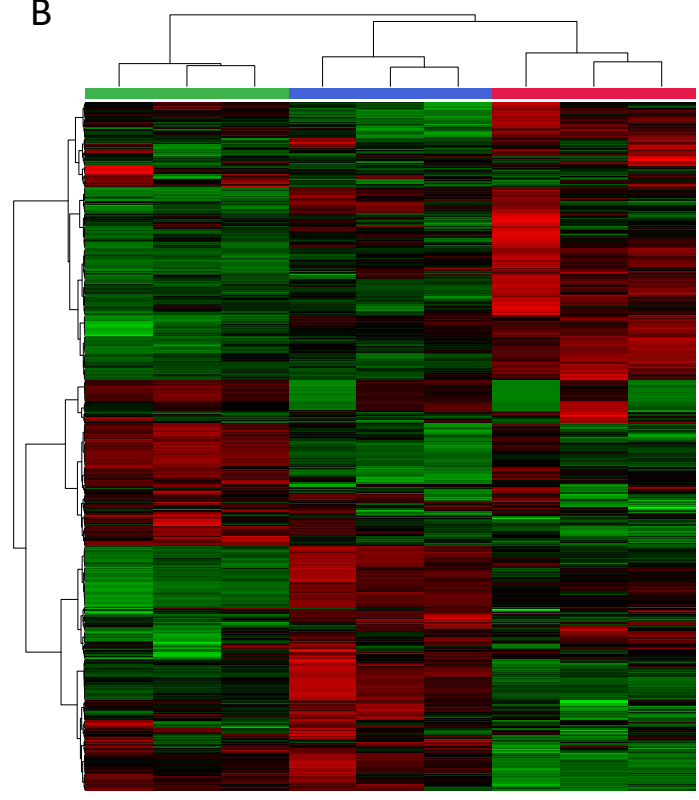

$\mathrm{D}$

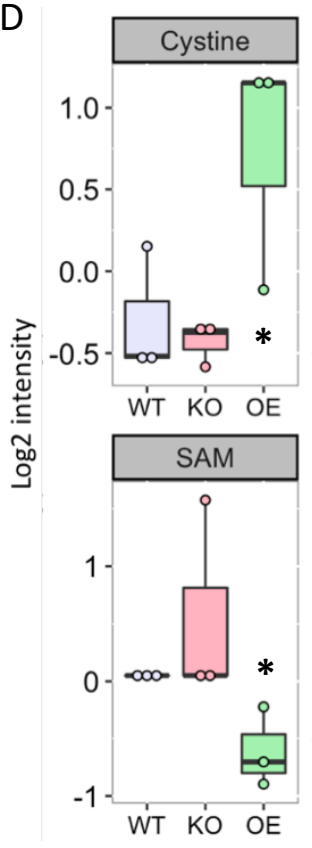

\begin{tabular}{l}
\hline FMO-2 OE \\
Wild type \\
FMO-2 KO
\end{tabular}

Figure 1: One carbon metabolism is altered by fmo-2 expression level. A) Principal component analysis of untargeted LC-MS metabolomics data of wild type, FMO-2 OE, and FMO-2 KO strains of C. elegans. B) Heatmap of untargeted LC-MS metabolomics data of the wild type, FMO-2 OE and FMO-2 KO. C) Pathway enrichment analysis using untargeted LC-MS metabolomics data of wild type and FMO-2 OE. D) Comparison of targeted metabolomics data of metabolites related to OCM between the wild type, FMO-2 $\mathrm{OE}$ and FMO-2 KO normalized to the median and log transformed. SAM = sadenosylmethionine. * represents $p<0.05$ using paired t-test. 

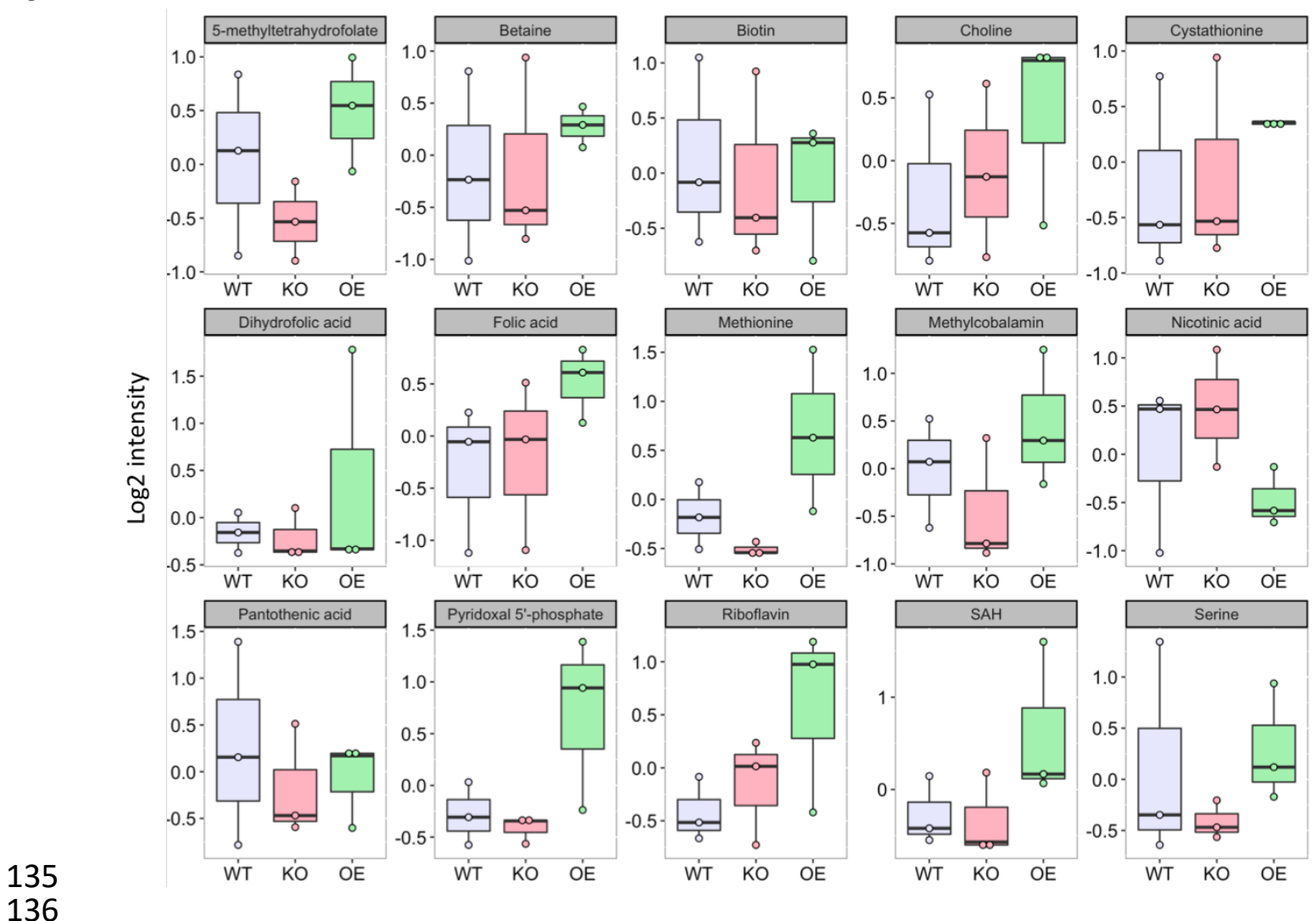

Supplementary Figure 1: Comparison of targeted metabolomics data of metabolites related to OCM between the wild-type, FMO-2 OE and FMO-2 KO. SAH = sadenosylhomocysteine. Data are median normalized and log transformed. 
One carbon metabolism interacts with fmo-2 to regulate stress resistance and longevity specifically, we next hypothesized that these metabolic changes are causal for longevity phenotypes. Previous studies identify increased stress resistance as a common phenotype shared by multiple long-lived organisms both within and between species ${ }^{24-27}$. To determine the

142 functional interaction between fmo-2 and OCM, we used RNAi to knockdown the expression of

143 genes involved in OCM (Figure 2A) and tested for their role in promoting or repressing survival

144 against the oxidative stressor paraquat. Individual knockdown of multiple genes exhibit altered

145 paraquat stress resistance phenotypes for the wild type and FMO-2 OE. Of the eight genes that

146 we tested, the knockdown of five genes, alh-3, atic-1, cbs-1, cth-2, and sams-1, abrogate FMO-

1472 OE resistance against paraquat (Figure 2B-F), as assessed using log-rank test with a cutoff

148 threshold of $p<0.0001^{28}$ compared to the empty vector (EV) controls. Our data for the

149 knockdowns of alh-3, atic-1, and cbs-1 are consistent with previous reports that their expression

150 levels are upregulated in long-lived worms ${ }^{11,29}$. alh-3 is upregulated in eat-2 mutants, atic-1 is

151 upregulated in both eat-2 and daf-2 mutants, and $c b s-1$ is upregulated under cold-induced

152 longevity and is required for the lifespan extension of eat-2 and glp-1 mutants ${ }^{11,29-31}$. cth-2 is a

153 homolog of the transsulfuration pathway enzyme cystathionine $\gamma$-lyase that is detrimental to the

154 wild type lifespan when its expression is suppressed using $\mathrm{RNAi}^{30}$. Thus, it is plausible that

155 these genes are required for multiple longevity pathways, including fmo-2-mediated longevity, to

156 confer resistance against paraquat. Interestingly, while sams-1 knockdown extends worm

157 lifespan ${ }^{17}$, we find that knocking down sams-1 abrogates FMO-2 OE paraquat resistance

158 (Figure 2F), suggesting that the regulation of lifespan and stress resistance are uncoupled in

159 this instance. This result is similar to previous work showing that sams-1 knockdown is

160 detrimental to survival under pathogen exposure ${ }^{32}$.

We also find that knocking down mel-32 increases stress resistance of both the wild type

162 and FMO-2 OE (Figure 2G), suggesting that the stress resistance conferred by the suppression 
163 of mel-32 is independent of fmo-2. The two remaining genes that we knocked down, $m$ trr- 1 and

164 Y106G6E.4, did not affect the stress resistance of the worms (Supplementary Figure 2).

165 Overall, our data show that five out of eight of the genes that we tested are required by FMO-2

166 OE to confer paraquat resistance. These results are consistent with the hypothesis that OCM is

167 a regulator of stress resistance and that there is a genetic interaction between fmo-2 and OCM

168 in that regulation. 
A

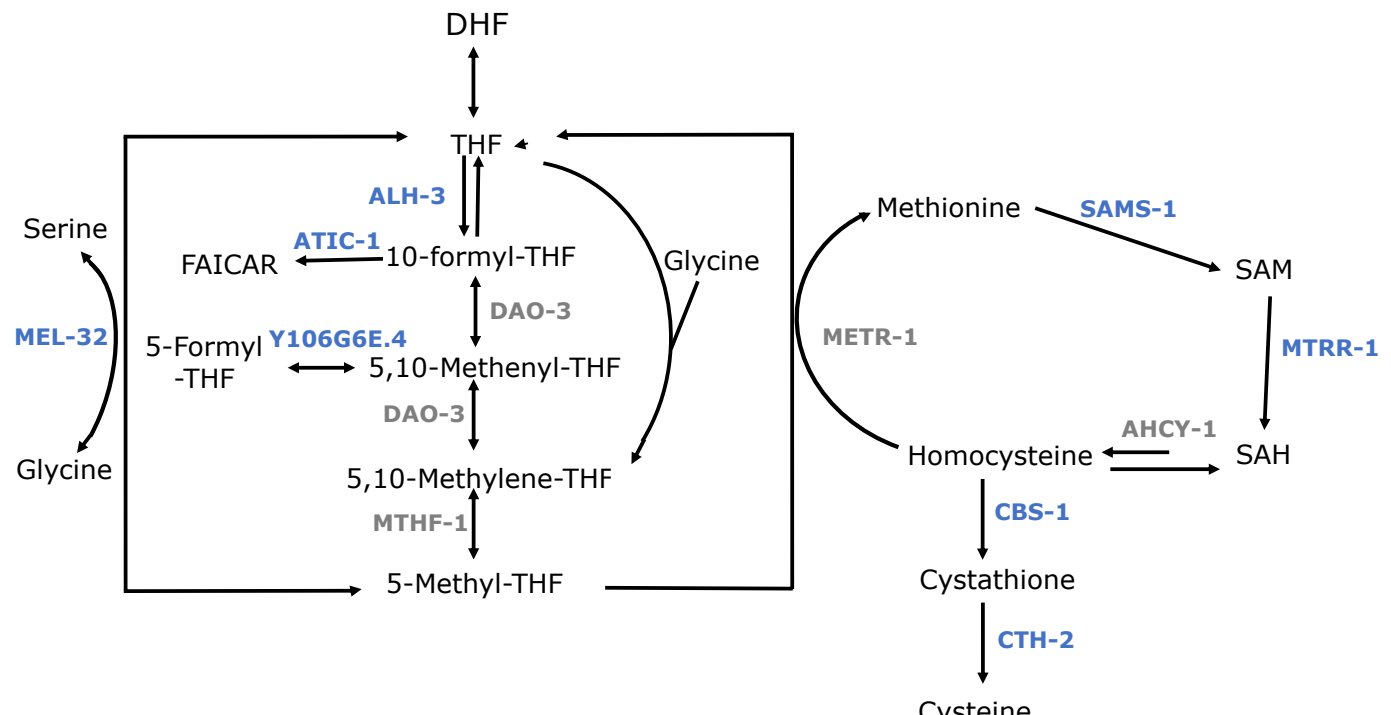

171

172

173

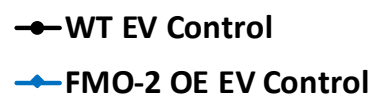

• •WT RNAi

- .FMO-2 OE RNAi

B

C
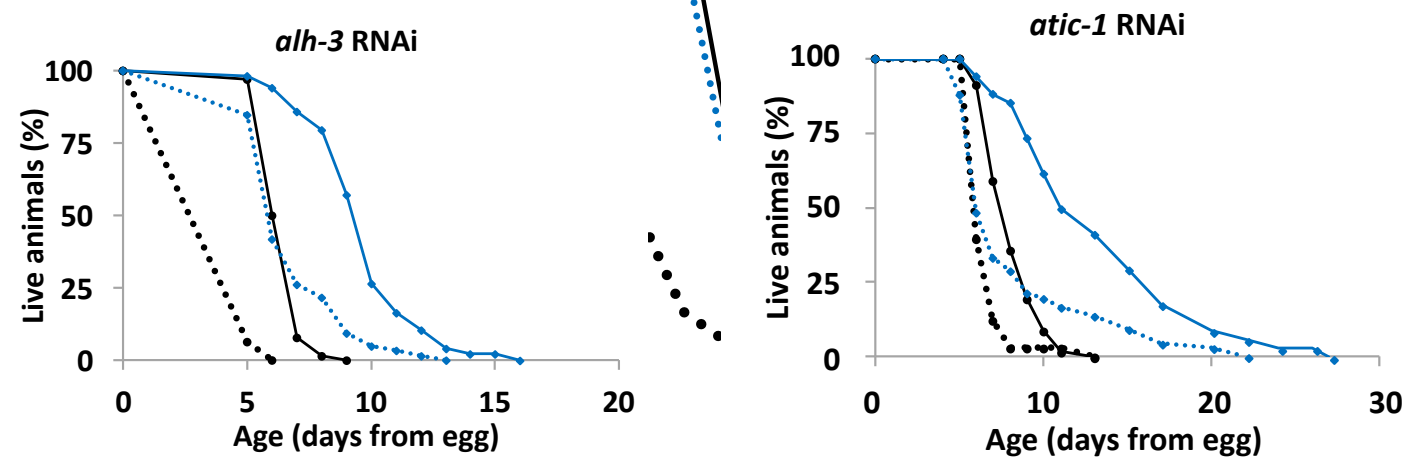

D

E
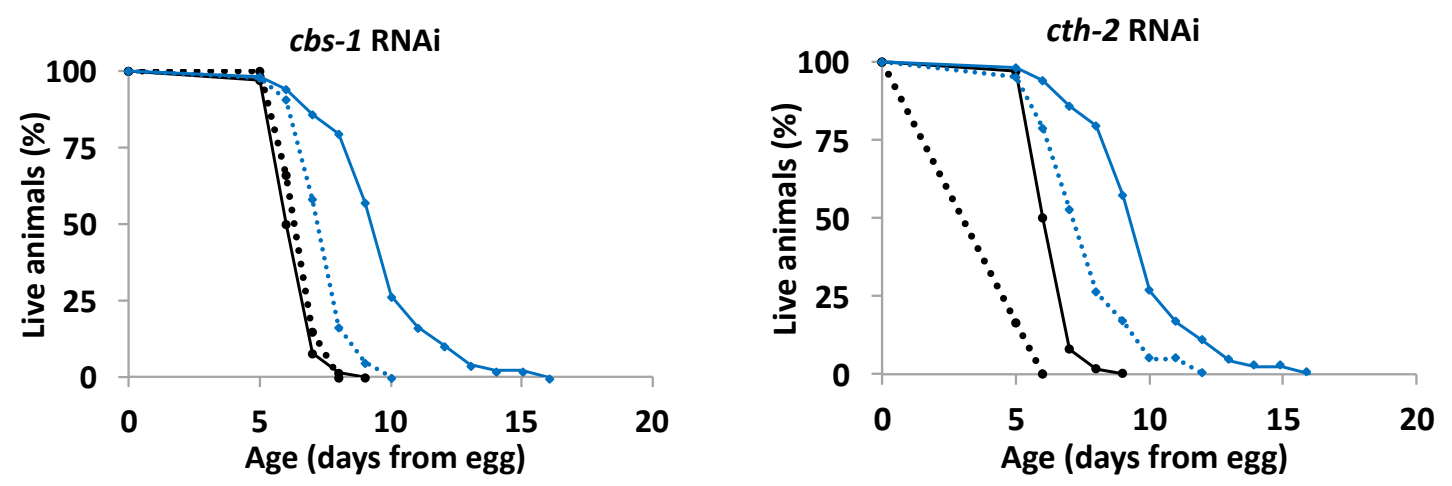
$\mathrm{F}$

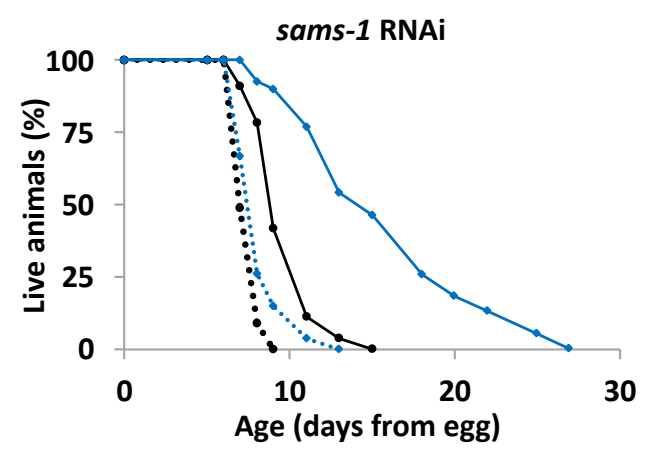

G

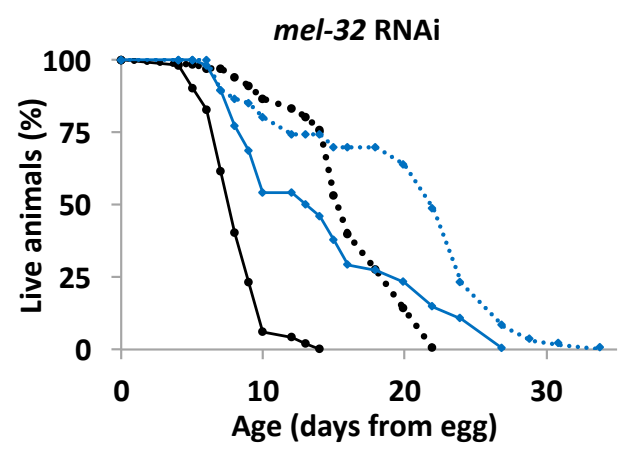

Figure 2: Fmo-2 interacts with OCM genes to regulate resistance against paraquat. A)

Diagram of OCM network. Genes included in the screen are labeled in blue and genes not included in the screen are labeled in gray. B-G) $5 \mathrm{mM}$ paraquat stress resistance assay (from L4) comparing the wild-type and FMO-2 OE on empty-vector (EV) and B) alh3 RNAi, C) atic-1 RNAi, D) cbs-1 RNAi, E) cth-2 RNAi, F) sams-1 RNAi, and G) mel-32 RNAi. Statistics in Supplementary Data 4. 
A

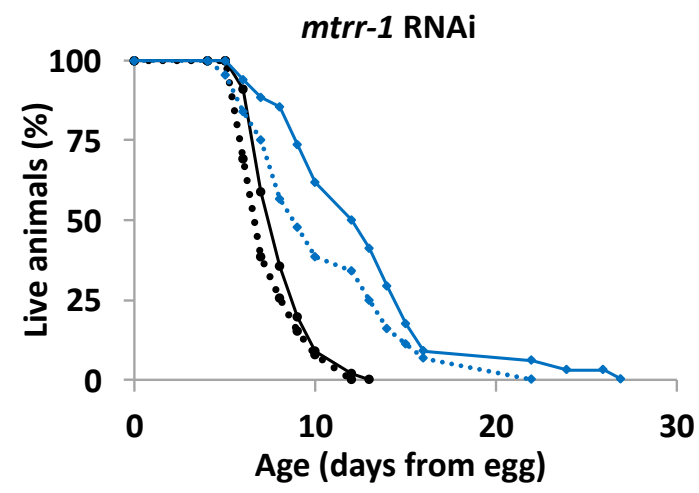

B

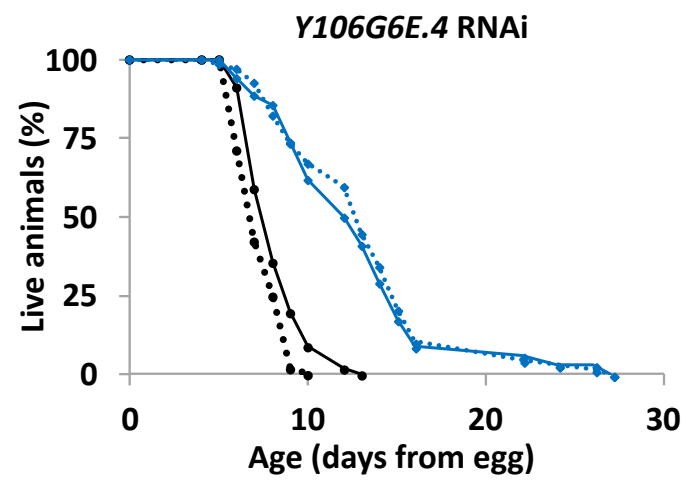

Supplementary Figure 2: OCM genes that do not alter worm stress resistance. $5 \mathrm{mM}$ paraquat stress resistance assay comparing the wild-type and FMO-2 OE on emptyvector (EV) and A) $m$ trr-1 RNAi and B) Y106G6E.4 RNAi. Statistics in Supplementary Data 4. 
To test the interaction between fmo-2 and OCM more directly, we measured the longevity of worms with RNAi knockdown of genes from our paraquat resistance screen. We included FMO-2 KO in the lifespan analysis to determine if the interactions that we identify are

191 dependent on fmo-2 expression. Similar to the paraquat survival assays, multiple gene

192 knockdowns showed altered lifespan phenotypes for the wild type, FMO-2 OE, and FMO-2 KO.

193 Of the eight genes we tested, knockdown of two genes, alh-3 and cth-2, suppress the lifespan

194 extension of FMO-2 OE to the level of the wild type (Figure 3A, B), as assessed using log-rank

195 test with a cutoff threshold of $p<0.0001^{28}$ compared to the empty vector (EV) controls,

196 consistent with our paraquat survival data. In contrast, knockdown of sams-1 increases the

197 lifespan of the wild type and FMO-2 KO to the level of FMO-2 OE without further extending the

198 lifespan of FMO-2 OE (Figure 3C), suggesting a separation between lifespan and stress

199 resistance. Additionally, we find that knocking down mel-32 only extends the lifespan of FMO-2

200 OE (Figure 3D). It is possible that the metabolic alterations caused by increased fmo-2

201 expression are required for mel-32 gene suppression to promote worm lifespan.

203 affect the lifespan of any of the worm strains (Supplementary Figure 3). Although knocking

204 down atic-1 and cbs-1 abrogated FMO-2 OE paraquat resistance (Figure 2C, D), they did not

205 abrogate FMO-2 OE lifespan (Supplementary Figure 3A, B). Similar to sams-1 knockdown

206 data, this finding suggests uncoupling of stress resistance and lifespan. In total, our data show

207 that two of the genes that we tested are required for FMO-2 OE lifespan extension, another

208 gene extends lifespan non-additively with FMO-2 OE, placing it in the same functional pathway,

209 and one of the genes only extends the lifespan of FMO-2 OE when knocked down. Thus, our

210 data support an interaction between fmo-2 and genes involved with OCM in regulating worm

211 lifespan. 
A

213

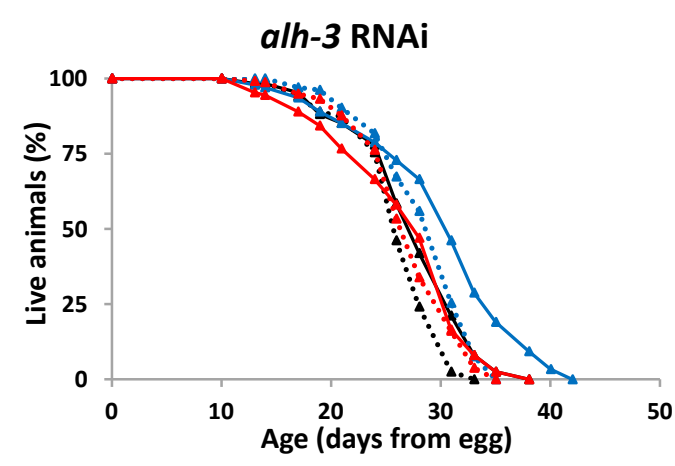

C

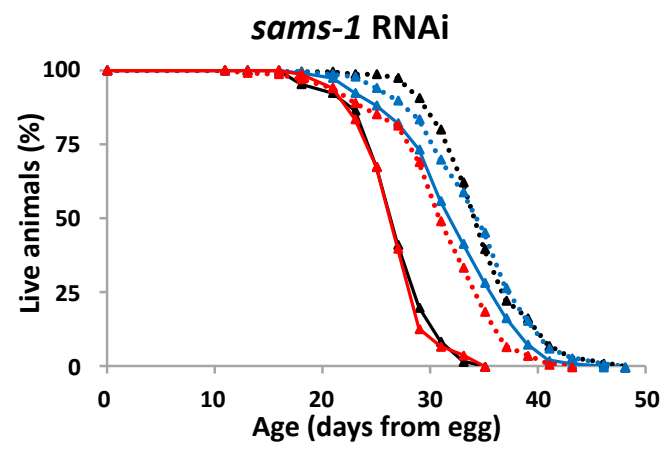

- FMO-2 KO RNAi

B

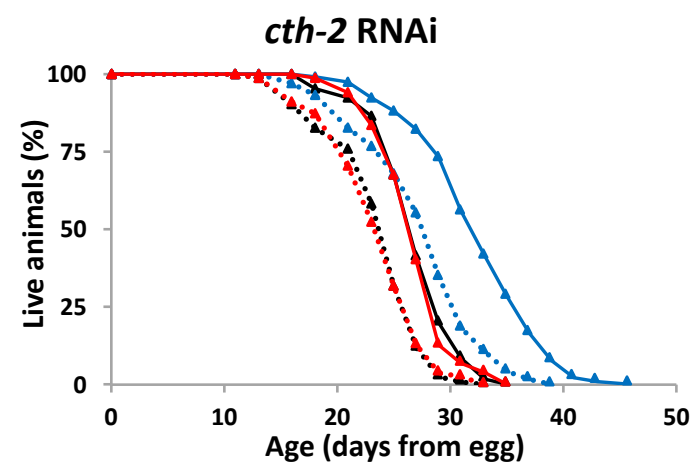

D

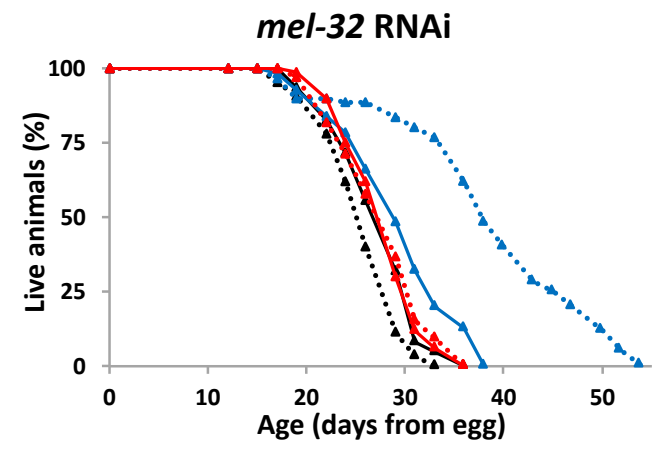

Figure 3: Fmo-2 interacts with OCM genes to regulate lifespan. Lifespan analysis comparing the wild-type and FMO-2 OE on empty-vector (EV) and A) alh-3 RNAi, B) cth-2 RNAi, C) sams-1 RNAi and D) mel-32 RNAi. Statistics in Supplementary Data 5. 
A

C
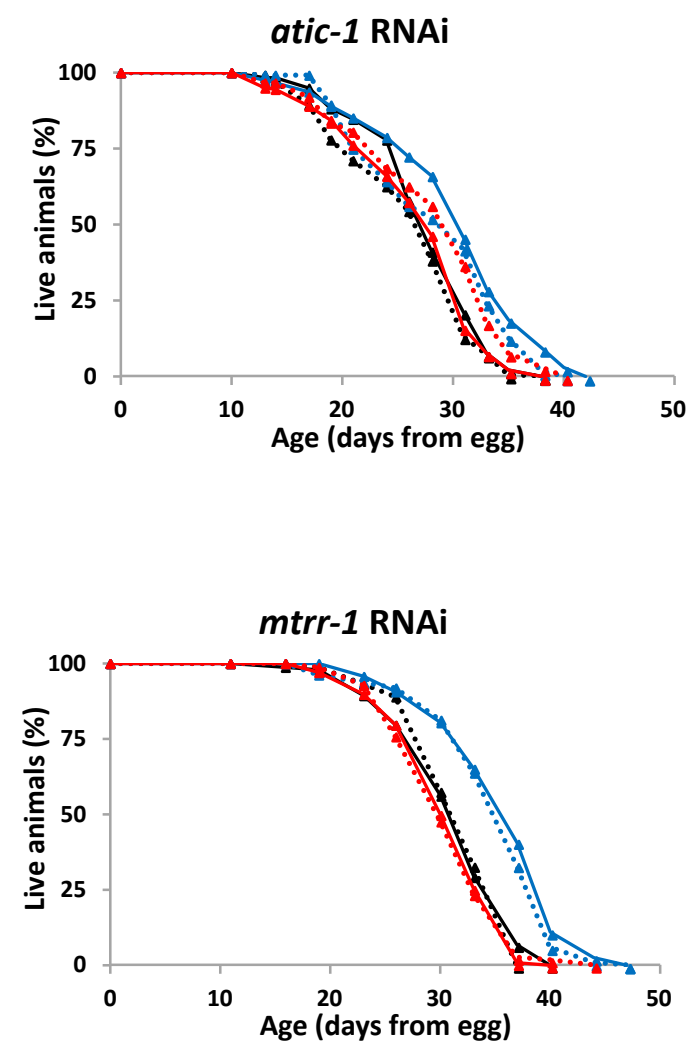

B

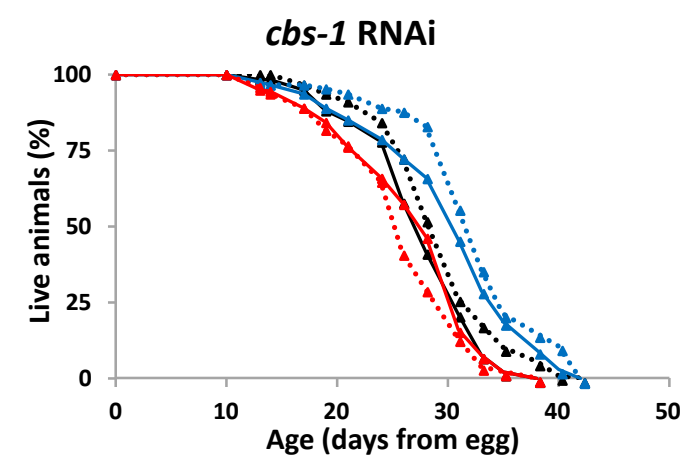

D

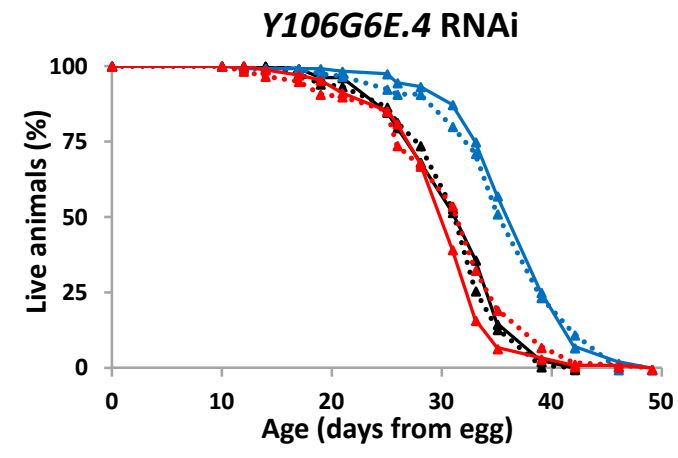

Supplementary Figure 3: OCM genes that do not alter worm lifespan. Lifespan analysis comparing the wild-type and FMO-2 OE on empty-vector (EV) and A) atic-1 RNAi, B) cbs1 RNAi, C) mtrr-1 RNAi, and D) Y106G6E.4 RNAi. Statistics in Supplementary Data 5. 
Fmo-2 influences longevity by modulating the transmethylation pathway

Our data are consistent with a model where fmo-2 interacts with OCM to regulate longevity and stress resistance. Previous studies identify multiple pathways that affect longevity and are also involved in OCM, including nucleotide metabolism, the transsulfuration pathway, and the transmethylation pathway ${ }^{11,16,17}$. Some of these pathways are also implicated in modifying longevity downstream of dietary restriction in multiple animal models ${ }^{16,17,33}$, making it

233 likely that one or more of these pathways are in the same functional pathway as fmo-2.

234 However, the metabolic consequences of fmo-2 expression on these pathways are not clear 235 based on the changes observed in our targeted metabolomics analysis alone, as the data only 236 show metabolic changes at a single time point and most of the metabolites within OCM are 237 intermediate metabolites. The stress resistance and lifespan results further complicate independent of fmo-2.

To help determine the biological relevance of the changes we observed in the OCM

241 network following fmo-2 expression, we applied a computational model (Supplementary Data

242 6) to predict how enzyme expression (Supplementary Data 7) changes may affect the output

243 fluxes of OCM. The model assumes a steady-state mass balance of fluxes in the reactions

244 illustrated in Figure 4A. This simple model includes eight reaction fluxes and five fluxes

245 representing transport of methionine (met), tetrahydrofolate (thf), s-adenosylmethionine (sam),

246 cysteine (cys), and 5,10-methylenetetrahydrofolate $(5,10$ thf) into and out of the folate cycle and

247 the methionine cycle. The model output fluxes represent important inputs for the nucleotide

248 metabolism, the transsulfuration pathway, and the transmethylation pathway, each of which are

249 reported to be important for influencing the aging process ${ }^{11,16,17}$ and are potential key targets for

250 the fmo-2-mediated longevity response. The stoichiometric coefficients for the reaction and

251 transport processes in this system are stored in the matrix S (Supplementary Data 8), where

252 under steady-state conditions $\mathbf{S}^{*} \mathbf{J}=\mathbf{0}$, where $\mathbf{J}$ is the vector of fluxes ${ }^{34,35}$. The entries in the 
vector $\mathbf{J}$ and matrix $\mathbf{S}$ are defined in Figure 4A. Vectors that satisfy the mass-balance

254 relationship $\mathbf{S}^{*} \mathbf{J}=\mathbf{0}$ are said to belong to the nullspace of $\mathbf{S}$. To predict how changes in the

255 expression of genes for the enzymes catalyzing the reactions in this network may affect the

256 output fluxes, we projected the gene expression data (Supplementary Data 7) onto the

257 nullspace of S (details are provided in the Methods). This projection predicts an inverse

258 correlation between fmo-2 expression and flux through methylation reactions, where the

259 methylation flux is predicted to be reduced in FMO-2 OE and increased in FMO-2 KO compared

260 to wild type (Figure 4B, Supplementary Figure 4). This analysis does not predict correlative

261 changes to flux through nucleotide metabolism or the transsulfuration pathway.

262 Based on this analysis, we hypothesized that artificially decreasing the flux through

263 methylation should replicate FMO-2 OE longevity in the wild type and FMO-2 KO strains, while

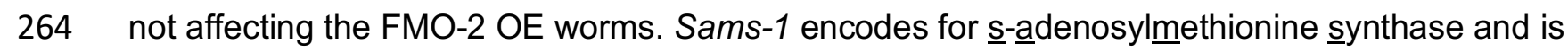

265 involved in the conversion of methionine into s-adenosylmethionine (SAM). Suppression of

266 sams-1 has been previously shown to decrease SAM level ${ }^{36}$ and increase longevity ${ }^{17}$. We find

267 that sams-1 RNAi recapitulates FMO-2 OE lifespan extension in the wild type and FMO-2 KO

268 worms while not affecting FMO-2 OE lifespan (Figure 3C). Our data are consistent with

269 previous studies showing that knockdown of sams-1 fails to further extend the lifespan of

270 genetic DR model eat-2 mutants ${ }^{17}$, thereby placing sams-1 knockdown in the same functional

271 pathway as FMO-2 OE.

To validate the model metabolically, we used the abundance level of SAM and s-

273 adenosylhomocysteine $(\mathrm{SAH})$ from our targeted metabolomics analysis to calculate the

274 SAM/SAH ratio. The SAM/SAH ratio is used as a biomarker for methylation potential, where a

275 decrease in the ratio suggests a hypomethylated state and an increase suggests a

276 hypermethylated state ${ }^{37,38}$. Consistent with our computational model prediction, we observed a

277 reduction in the SAM/SAH ratio for FMO-2 OE (hypomethylation) and an increase in the ratio for

278 FMO-2 KO (hypermethylation) compared to the wild type (Figure 4C). Overall, our 
bioRxiv preprint doi: https://doi.org/10.1101/2021.06.18.449022; this version posted June 18, 2021. The copyright holder for this preprint (which was not certified by peer review) is the author/funder. All rights reserved. No reuse allowed without permission.

279 computational model prediction and experimental data support the hypothesis that fmo-2

280 expression reduces flux through the transmethylation pathway, and that this reduction extends

281 worm lifespan. 
283

284

285

286

287

288

289

290

291

292

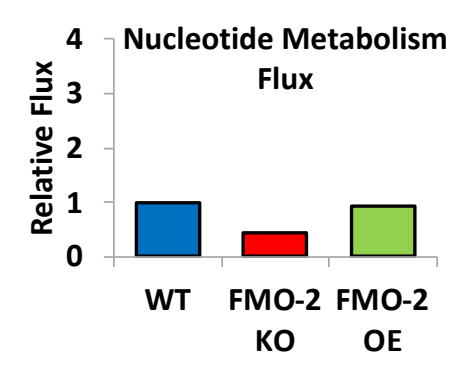

C

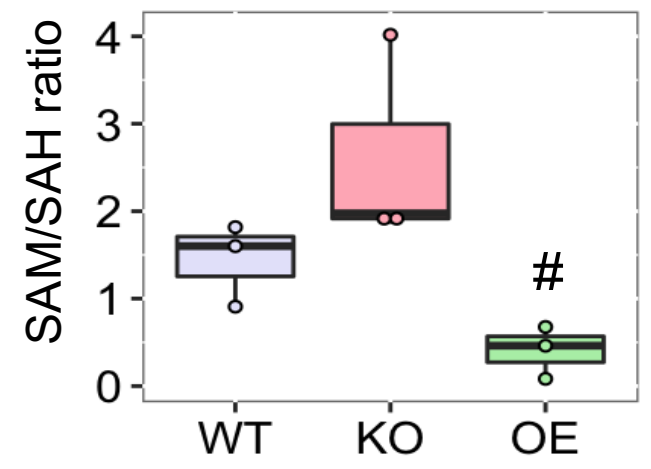

B
Flux Vector (J) Zero Vector (0)

A

\section{Stoichiometric Matrix (S)}

$\begin{array}{lllllllllllll}\text { R1 } & \text { R2 } & \text { R3 } & \text { R4 } & \text { R5 } & \text { R6 } & \text { R7 } & \text { R8 } & \text { R9 } & \text { R10 } & \text { R11 } & \text { R12 } & \text { R13 }\end{array}$

\begin{tabular}{|c|c|c|c|c|c|c|c|c|c|c|c|c|c|}
\hline met & -1 & 0 & 0 & 1 & 0 & 0 & 0 & 0 & 1 & 0 & 0 & 0 & 0 \\
\hline sam & 1 & -1 & 0 & 0 & 0 & 0 & 0 & 0 & 0 & 0 & -1 & 0 & 0 \\
\hline sah & 0 & 1 & -1 & 0 & 0 & 0 & 0 & 0 & 0 & 0 & 0 & 0 & 0 \\
\hline hcy & 0 & 0 & 1 & -1 & -1 & 0 & 0 & 0 & 0 & 0 & 0 & 0 & 0 \\
\hline cyst & 0 & 0 & 0 & 0 & 1 & -1 & 0 & 0 & 0 & 0 & 0 & 0 & 0 \\
\hline cys & 0 & 0 & 0 & 0 & 0 & 1 & 0 & 0 & 0 & 0 & 0 & -1 & 0 \\
\hline th & 0 & 0 & 0 & 1 & 0 & 0 & -1 & 0 & 0 & 1 & 0 & 0 & 0 \\
\hline 5,10 thf & 0 & 0 & 0 & 0 & 0 & 0 & 1 & -1 & 0 & 0 & 0 & 0 & -1 \\
\hline $5 \mathrm{mthf}$ & 0 & 0 & 0 & -1 & 0 & 0 & 0 & 1 & 0 & 0 & 0 & 0 & 0 \\
\hline
\end{tabular}
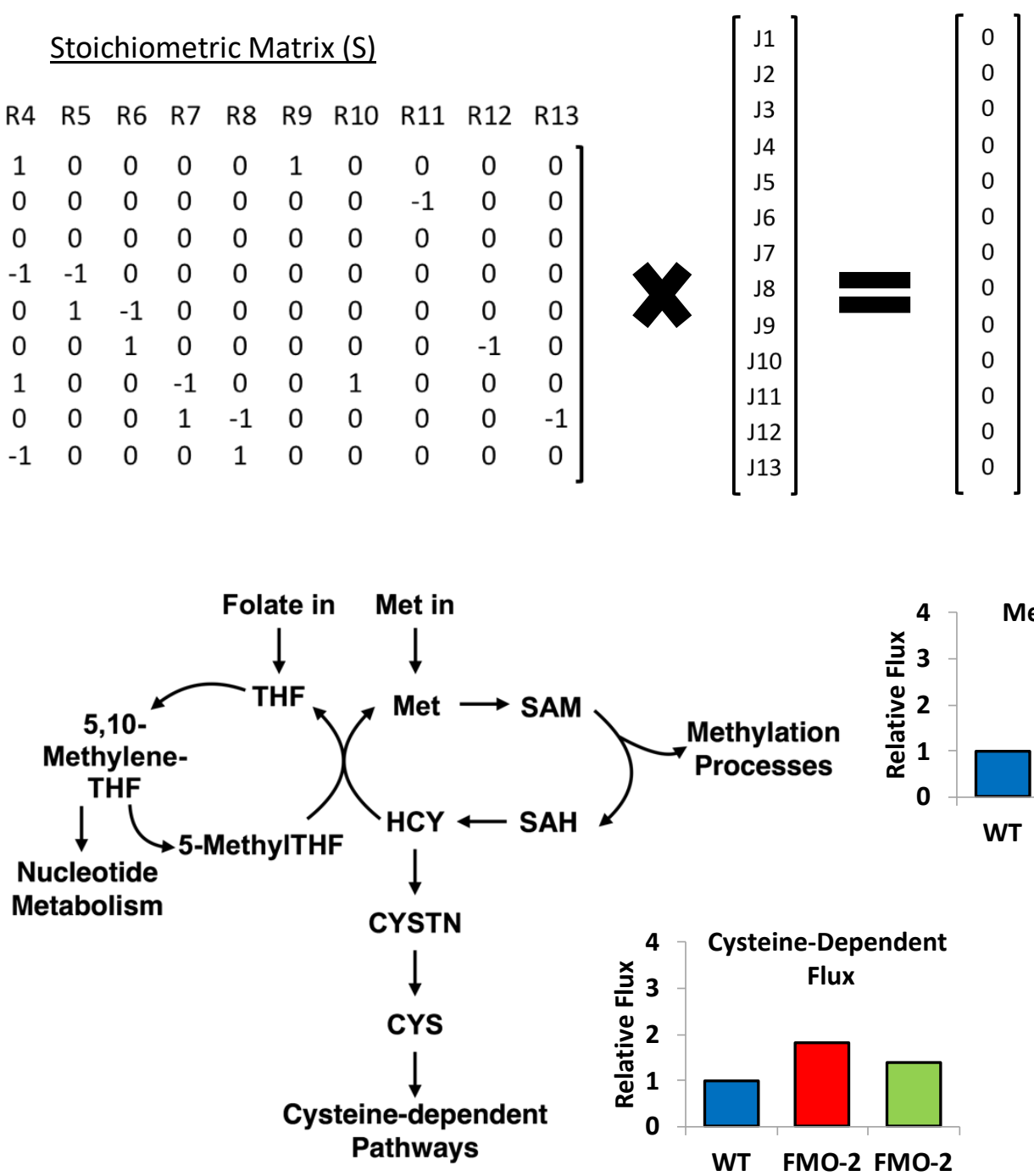
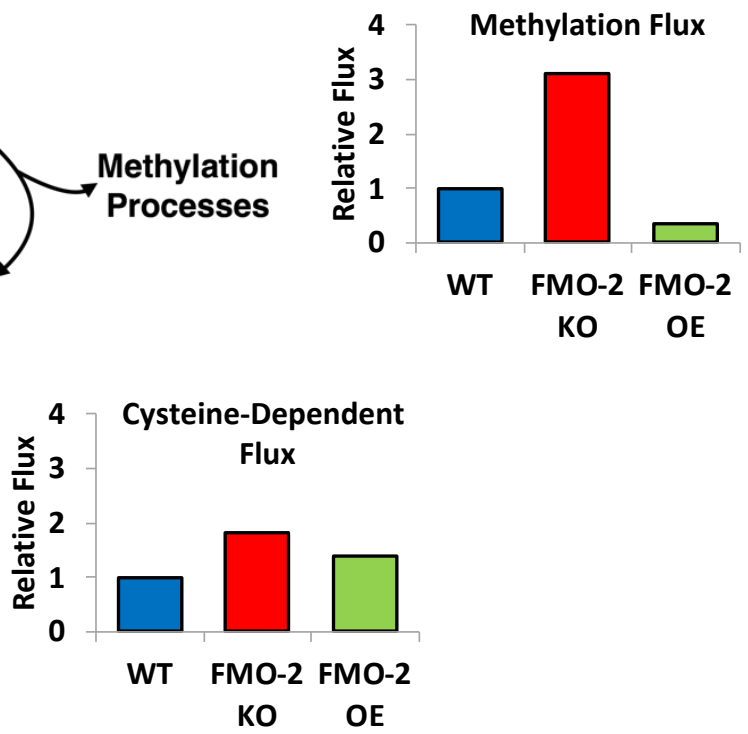

Figure 4: Methylation flux is altered following changes in fmo-2 expression. A) Schematic of computational model. B) Model predictions of output metabolic fluxes. C) SAM/SAH ratio of the wild-type (WT), FMO-2 OE (OE) and FMO-2 $\mathrm{KO}(\mathrm{KO})$. \# = p < 0.05 compared to the wild-type using one-way ANOVA. 


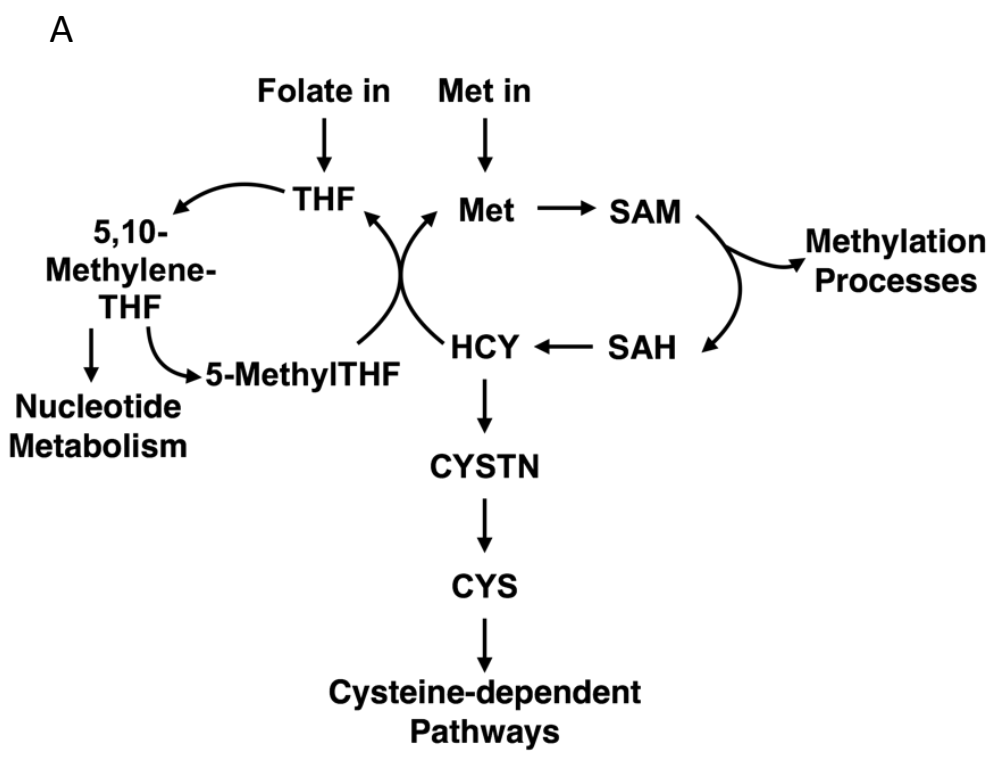

B

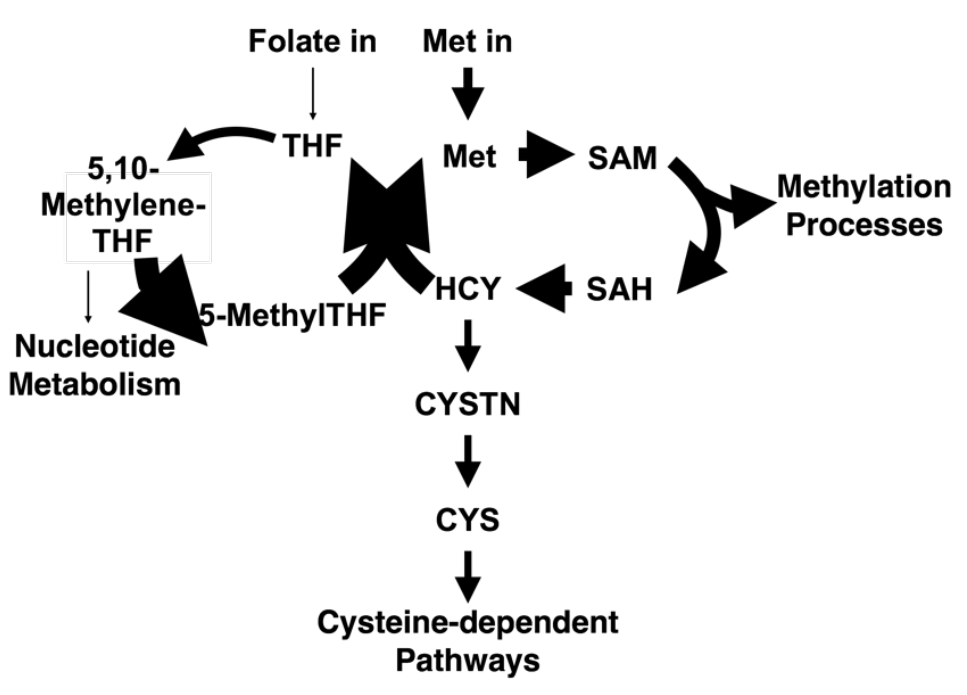

C

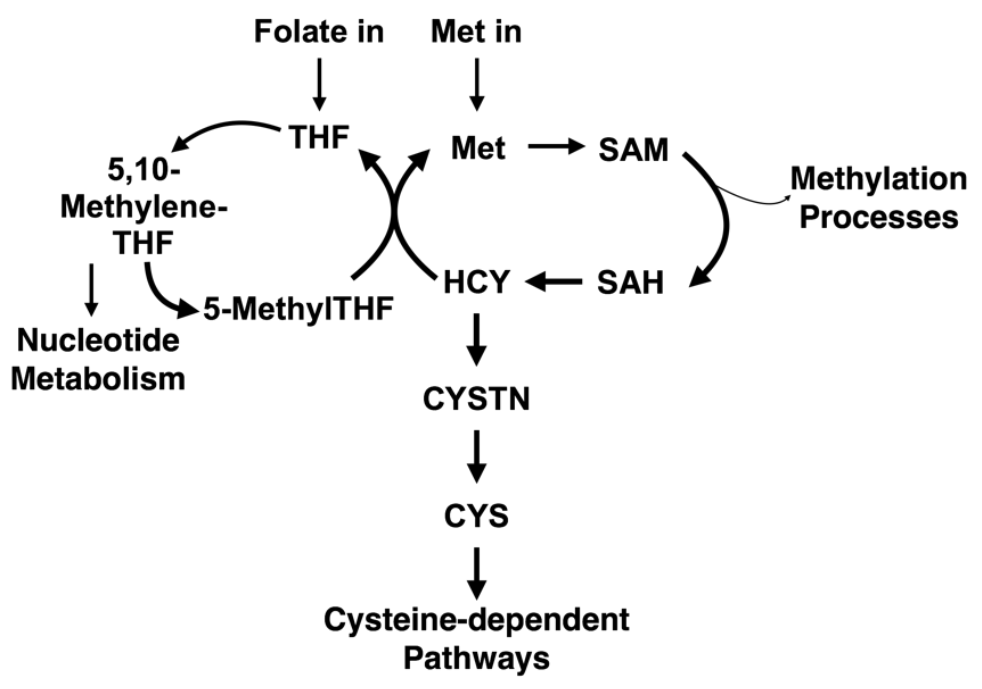

Supplementary Figure 4: Computational model predicts reduced flux through methylation processes. Model predictions of OCM fluxes for A) the wild-type, B) FMO-2 OE, and C) FMO$2 \mathrm{KO}$ after normalization to the wild-type. Arrow weights represent changes in each flux relative to the wild type, which is set to be equal to 1. 
Mammalian FMO metabolomics analysis reveals tryptophan as a substrate of FMO-2

Our data thus far suggest a model where fmo-2 interacts with OCM to modulate the aging process. However, since FMOs are promiscuous enzymes that oxygenate many nucleophilic atoms, the mechanism by which fmo-2 induction leads to changes in OCM is not readily evident. FMOs are known as xenobiotic metabolizing enzymes, with many known exogenous targets and few known endogenous targets ${ }^{1}$. Despite extensive knowledge on their enzymatic activity and recent data linking FMOs to endogenous metabolism, no link between specific and systemic metabolism has been made. We hypothesize that a limited number of FMO targets are causal in FMO-2's effects on OCM and, importantly, on the aging process.

CeFMO-2 (Figure 5A), we referred to our previously published targeted metabolomics of mouse FMO overexpressing (OE) HepG2 cells to determine potential metabolic targets of FMO-2 ${ }^{10}$.

307 Our selection criteria for putative substrates of FMO-2 included identifying metabolites that had decreased abundance in at least three of the five FMO OE cell lines to pDEST controls. We use this stringent criteria to identify the most well-conserved targets of FMOs, given that no data exist for CeFMO-2 targets. Using this approach, we identified tryptophan and phenylalanine as

311 potential substrates of FMOs (Figure 5B). To determine if either of these are substrates of

312 FMO-2, we measured the enzymatic activity of isolated FMO-2 protein in the presence of 313 varying concentrations of tryptophan and phenylalanine. We find that FMO-2 is active toward 314 tryptophan at a reasonable $\mathrm{K}_{\mathrm{m}}$ and $\mathrm{k}_{\text {cat }}\left(\mathrm{K}_{\mathrm{m}}: 880 \pm 430 \mu \mathrm{M} ; \mathrm{K}_{\text {cat. }}: 9.7 \pm 1.5 \mathrm{sec}^{-1}\right)$, suggesting that 315 tryptophan is a viable substrate of FMO-2 (Figure 5C, Supplementary Data 9). FMO-2 was 316 also active toward phenylalanine, but enzymatic activity did not become apparent until $10 \mathrm{mM}$, 317 suggesting that phenylalanine is not likely a good endogenous substrate of FMO-2 (Figure 5D).

318 Since FMO-2 has no previously reported activity toward tryptophan, we used LC-MS with 100, 319250 , and $500 \mu \mathrm{M}$ tryptophan under the same enzymatic conditions to determine the product of 320 tryptophan oxygenation. Our resulting data show the presence of $\mathrm{N}$-formylkynurenine in a 
321 concentration dependent manner in each of the samples, suggesting that is the product formed

322 by FMO-2 activity toward tryptophan (Figure $5 \mathrm{E}$ ). To determine whether tryptophan is a

323 conserved substrate of FMOs, we next tested whether mFMO5 can also oxygenate tryptophan.

324 mFMO5 also shows increased levels of N-formylkynurenine based on HPLC analysis under

325 tryptophan enzymatic conditions (Figure 5E, Supplementary Data 10). Alignment of mFMO5

326 and CeFMO-2 using ancient mammalian FMO5 ${ }^{39-41}$ shows that all but one of the catalytic

327 residues of CeFMO-2 are conserved in mFMO5 (Figure 5A), so it follows that they would have

328 similar activity toward some substrates. The kinetic parameters of FMO-2 toward NADPH,

329 methimazole, and tryptophan are summarized in Figure 5F. The poor substrates (such as

330 cysteine, phenylalanine, and TMA) and non-substrates of FMO-2 (such as 2-heptanone) are

331 summarized with either the concentration of substrate at which FMO-2 activity is first detected

332 or labeled not determined (N.D.) in Supplementary Data 9.

333 Based on our initial data linking FMO-2 to OCM, it is important to note that in addition to

334 being a key process in the kynurenine pathway, the conversion of tryptophan to N-

335 formylkynurenine precedes the conversion of $\mathrm{N}$-formylkynurenine to kynurenine by

336 formamidase, a process that releases formate, which is also a carbon source for $\mathrm{OCM}^{42}$.

337 Formate can enter OCM through the folate cycle, thus providing a connection between

338 tryptophan metabolism, the kynurenine pathway, and OCM. Based on this information, we

339 hypothesize that the kynurenine pathway is a target of FMO-2 that leads to changes in OCM. 


\section{$\mathrm{H} \mathrm{H} \mathrm{T}$}

$\mathrm{HH}$ A

QFL

F L T

$\mathrm{H} \mathrm{HI}$

$\mathrm{HH}$

TNT S

INTS

$\underline{\mathrm{H} H \mathrm{~T}}$

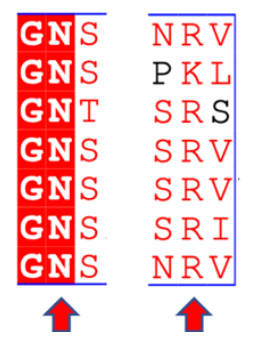

$\mathrm{QH}$

$\mathrm{A} \mathrm{HP}$

P KF

REP

$K E P$

$\mathrm{K} E \mathrm{P}$

Q H

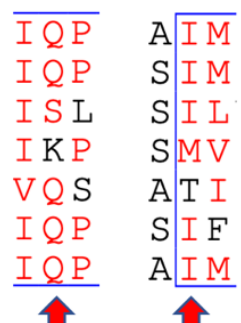

공 1

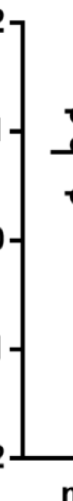

Tryptophan

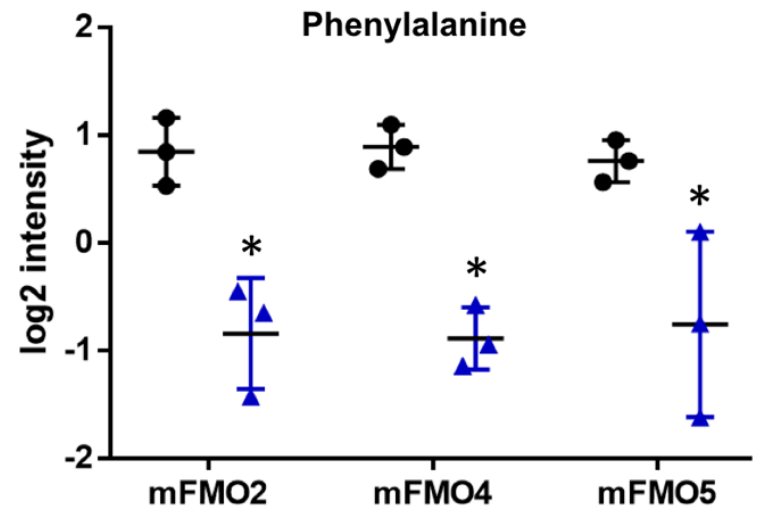

- pDEST control $\Delta \mathrm{mFMO} O \mathrm{OE}$

C

FMO-2 Enzyme Activity

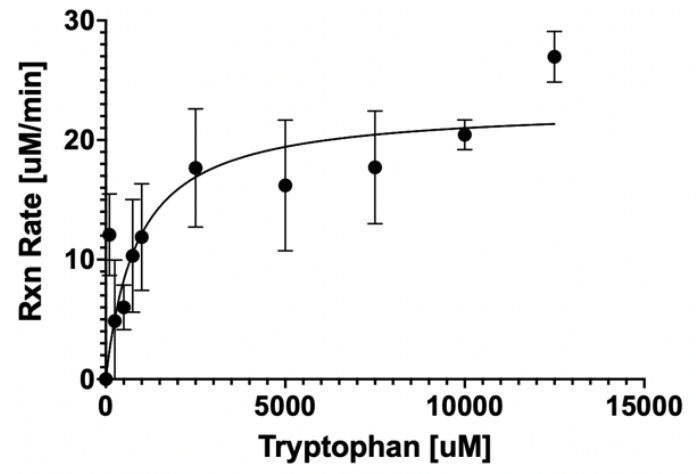

E N-formylkynurenine Product Formation

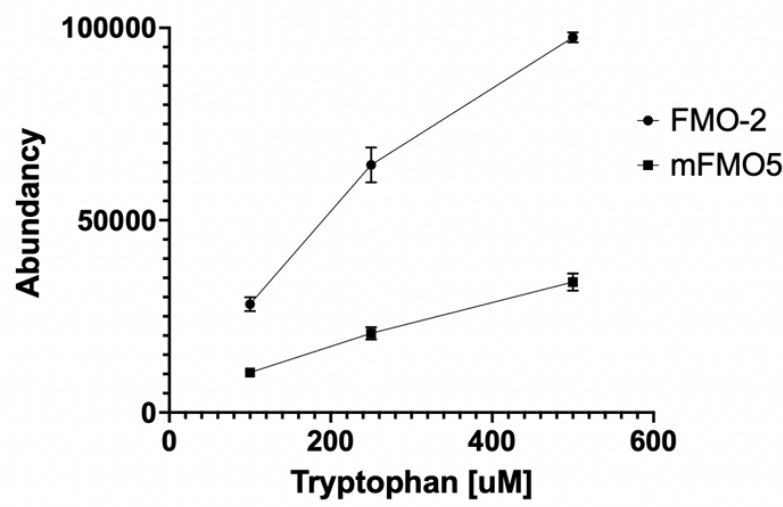

D

FMO-2 Enzyme Activity

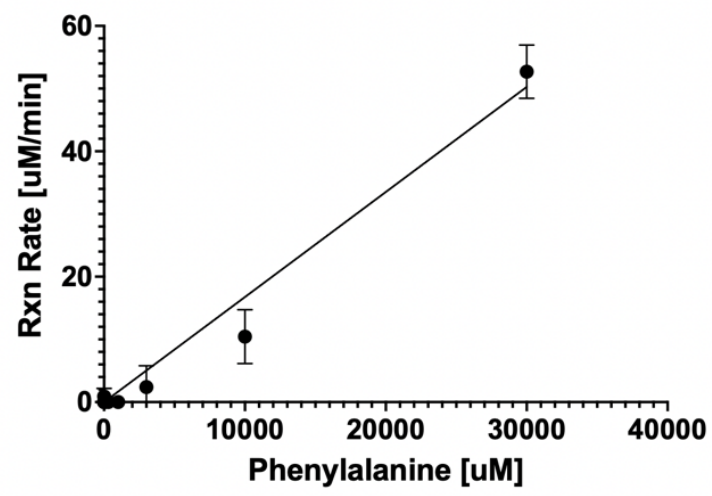




\begin{tabular}{|c|c|c|c|}
\hline Substrate & $\mathbf{K}_{\mathbf{m}}(\mathbf{m M})$ & $\mathbf{k}_{\text {cat }}$ (sec $\left.\mathbf{- 1}\right)$ & $\begin{array}{c}\text { Catalytic Efficiency } \\
\left(\mathbf{s e c}^{-1} \mathbf{~ M}^{-\mathbf{1}}\right)\end{array}$ \\
\hline NADPH & $2.50 \pm 1.24$ & $264 \pm 98$ & $105000 \pm 65000$ \\
\hline Methimazole & $1.92 \pm 1.14$ & $13.0 \pm 6.0$ & $6800 \pm 5100$ \\
\hline Tryptophan & $0.88 \pm 0.43$ & $9.7 \pm 1.5$ & $11000 \pm 5000$ \\
\hline
\end{tabular}

Figure 5: Mammalian FMO metabolomics analysis reveals the tryptophan/kynurenine pathway as a target of FMO-2. A) Conserved catalytic residues between CeFMO-2 and mouse FMOs (indicated by red arrows). B) The level of phenylalanine and tryptophan present in HepG2 cells expressing pDEST control vector, $\mathrm{mFMO2,} \mathrm{mFMO4}$, and mFMO5. * represents $p<0.05$ by paired t-test. $C$-D) The reaction rate by concentration for purified CeFMO-2 enzyme toward tryptophan and phenylalanine at $30^{\circ} \mathrm{C}$. E) The abundancy of $\mathrm{N}-$ formylkynurenine based on HPLC analysis of CeFMO-2 and mFMO5 activity toward 100, 250 , and $500 \mathrm{uM}$ tryptophan at $30^{\circ} \mathrm{C}$. F) Summary table of Michaelis-Menton parameters for CeFMO-2 cofactor and substrates.

AnCFMO5

AncFMO5

C.elegans_FMO-2 Mouse_FMO4 Mouse_FMO1

Mouse_FMO3

Mouse_FMO2

Mouse_FMO5

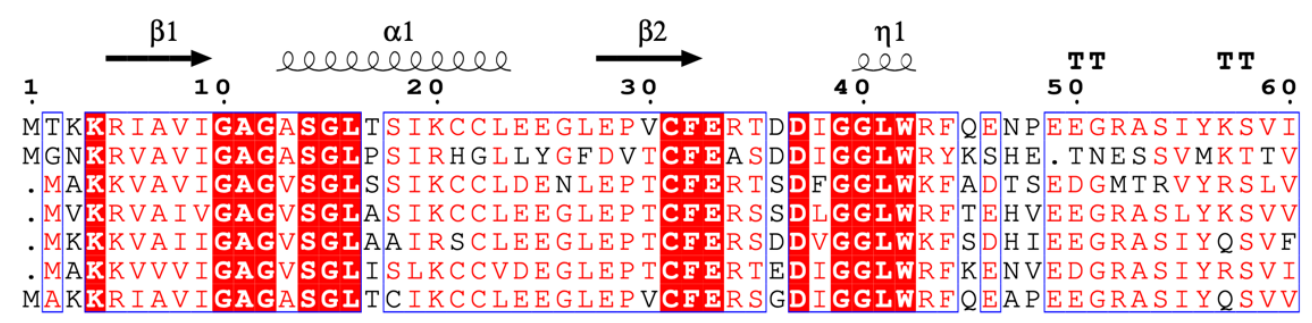

AnCFMO5

AnCFMO5

C.elegans_FMO-2

Mouse_FMO4

Mouse FMO1

Mouse_FMO3

Mouse_FMO2

Mouse_FMO5

$\alpha 2$

ele $_{70}$ т T

T T

$\alpha 3$

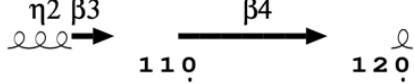

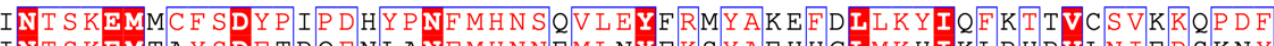

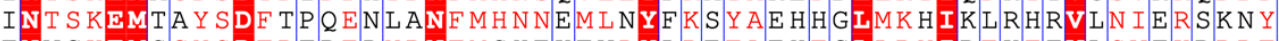
TNVCKEMSCYSDFP F REDYPNFMSHEKFWDYLREFAEHFGLLRYIRFKT TVLSVTKRP DF

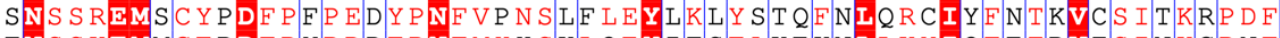

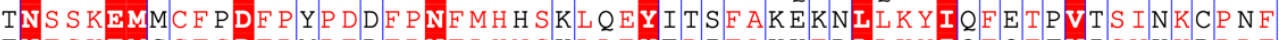

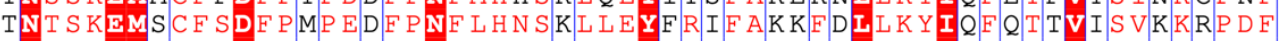

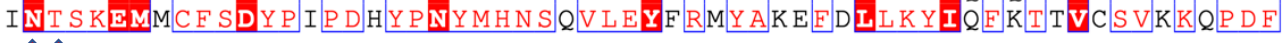
11

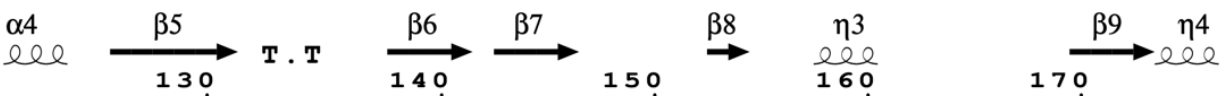

ST SGQWEVVTE C . GKKEVDVFDGVMVCTGHHT NAHLPLESFPGIEKFKGQYFHSRDYKN DNDGTWKVIYQTPEEKTLEEIFDGVLVCSGHHAIPHWPK. PFPGQNEFKGR IVHSHDYKD

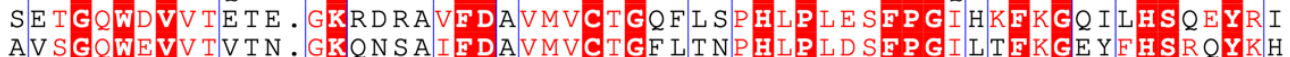
AVSGQWEVVTVTN. GKQN S A IT DAVMVCTGF L T NPHLPLD SFPGI L T F K GEY F H S R Q Y K H AS S GQWEVYTQS N. GKEQRTVEDAVMVCSGH I QPHLPLKSFPGIERTRGQYFHSREYKH

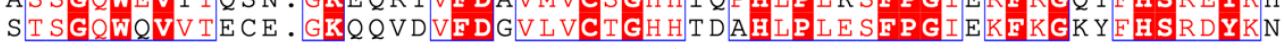


bioRxiv preprint doi: https://doi.org/10.1101/2021.06.18.449022; this version posted June 18, 2021. The copyright holder for this preprint (which was not certified by peer review) is the author/funder. All rights reserved. No reuse allowed without permission.

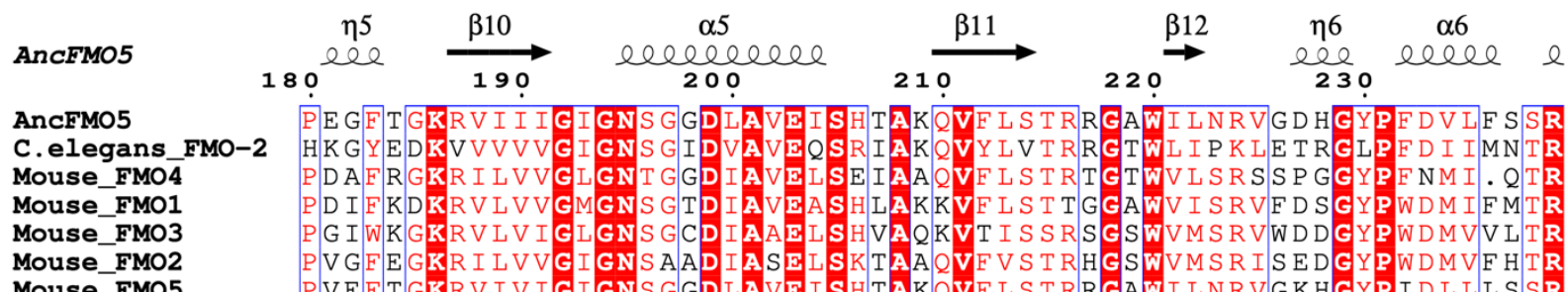

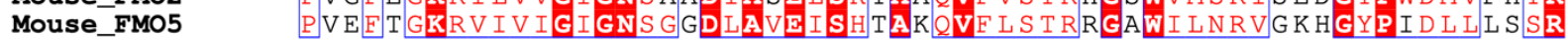

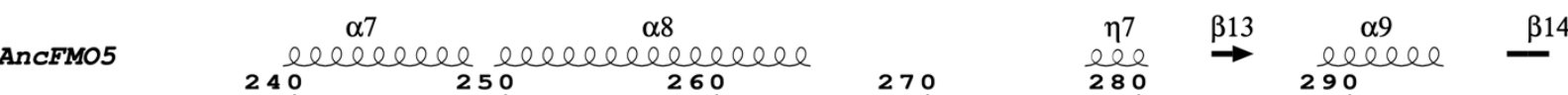

AnCFMO5

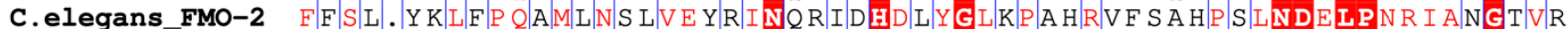

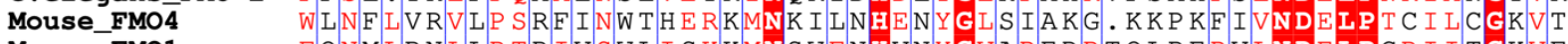
Mouse_FMO1 F

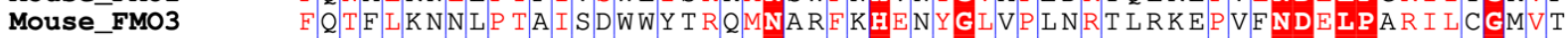
Mouse_FMO2 F S S M L R N V L P R T V V V K WMME Q Q MN R W F N H E N Y G L V P Q N K Y Y L M K E P V V L NDD D L P S R L L Y G A I K

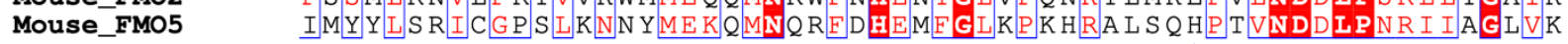

AnCFMO5

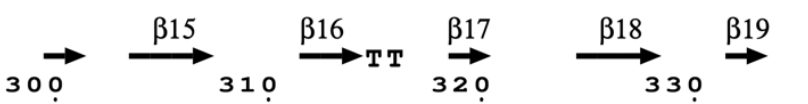

$\eta 8$
340

$\underset{350}{\rightarrow} \underset{\mathrm{T}}{\stackrel{\beta 2}{\rightarrow}}$

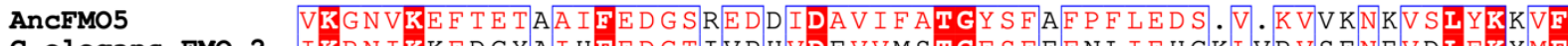

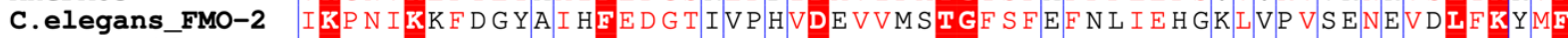
Mouse FMO4 Mouse FMO1 Mouse_FMO3 Mouse_FMO2 Mouse_FMO5

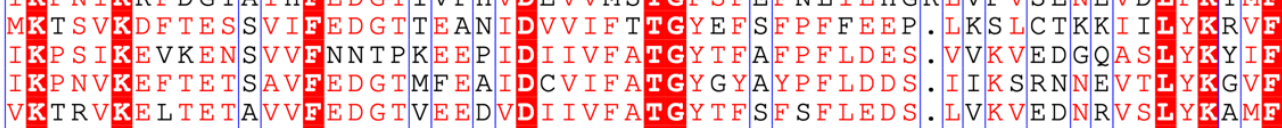

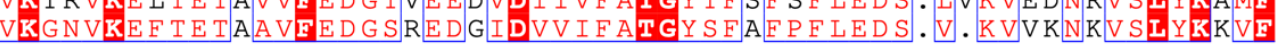

AnCFMO5

AncFMO5 C.elegans_FMO-2 Mouse_FMO4 Mouse_FMO1 Mouse FMO3 Mouse FMO2 Mouse_FMO5 $\stackrel{\beta 22}{\longrightarrow} \stackrel{\beta 23}{\longrightarrow}$ eleceleele

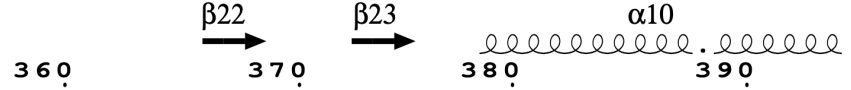
I Q P L GA I MP I S E L Q QRV . AT Q V F KG

RW. AT QVF KGLKT LP

400

$\alpha 11$

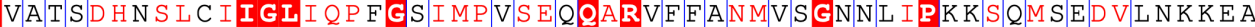
PPNI. ERA T LA I I GI I S LNGSI LVGT EF QARW. A T R VFKGLCS IPP S QKL MAE AT K T E Q I PAHL.PKPTLAVIGLIKP L GSMVPT GE T QARW.VVVVLKGATT LPPP SVMMEEVNERKKN

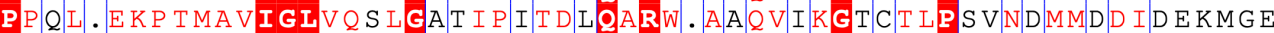

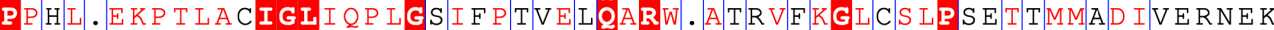

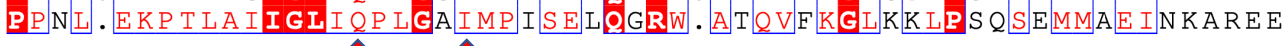


AnCFMO5

AnCFMO5

C.elegans_FMO-2

Mouse_FMO4

Mouse_FMO1

Mouse_FMO3

Mouse FMO2

Mouse_FMO5

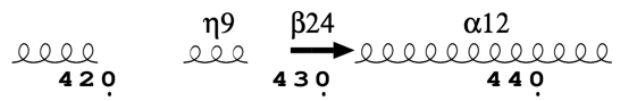

$\alpha 12$ MAKR|Y VD

ele $\overrightarrow{430}$ elebelobe

$\alpha 13$

450

$\alpha 13$

$\alpha 14$
elelelel n10 IKRG V V S RR H T I QV V D Y I P Y M D E L A E L I G C Q V P I L R T L F T D P V L G L R L F F GPNA G Y C Y RI KH S GF G L C Y C KA L Q T D Y I T Y I D D L L T S I N A K P D L RAML L TDPRIA LS I F F GPC T P Y H F R L

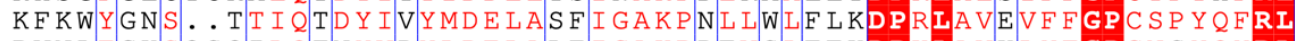

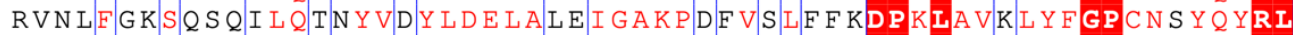
MAKRYVDSQRHT I GDY IDTMEE I AD LVGVRPNI LP LVF TDPRLA LR L L LGPC T P V Q YRL elegans FMO-2. Arrow heads indicate catalytic residues as determined by the crystal structure of ancestral mammalian FMO5 solved by Nicoll, et al. Overall identity of murine FMO5 and $C$. elegans FMO-2 is approximately $43 \%$. Alignment figures were generated using Clustal Omega and ESPript 3.0. 
To test this hypothesis, we assessed whether genes involved in tryptophan metabolism interact epistatically with FMO-2 (Figure 6A). Like our RNAi analyses of the OCM genes, we observed changes in stress resistance of the wild type and FMO-2 OE following the knockdown of genes involved in the kynurenine pathway (Figures 6B, C). Similarly, we observed changes in the lifespan of the wild type, FMO-2 OE, and FMO-2 KO worms under the same conditions

(Figures 6D, E), as assessed using log-rank test with a cutoff threshold of $p<0.0001^{28}$ compared to the empty vector (EV) controls. Here, we again observed a separation between the regulation of stress resistance and lifespan under kmo-1 and tdo-2 knockdown (Figures 6B-E).

371 Knocking down kmo-1 increases the resistance to paraquat in the wild type (Figure 6B), but it decreases the lifespan of the wild type, FMO-2 OE, and FMO-2 KO (Figure 6D). These data suggest that kmo-1 knockdown may be beneficial for resistance against paraquat, but that kmo1 expression is necessary for normal worm longevity. Knocking down tdo-2 abrogates paraquat resistance in FMO-2 OE (Figure 6C), but extends the lifespan of the wild type, FMO-2 OE, and FMO-2 KO (Figure 6E). Tdo-2 knockdown was previously reported to extend lifespan by inhibiting tryptophan degradation and thereby improving the regulation of proteotoxicity ${ }^{19}$. In addition, knocking down kynu-1 did not affect paraquat resistance of the worms (Supplementary Figure 6A), but it partially recapitulated the FMO-2 OE lifespan phenotype in the wild type and FMO-2 KO without affecting FMO-2 OE lifespan (Figure 6F), consistent with kynu-1 functioning in the same pathway as fmo-2. The knockdown of afmd-1 did not affect the stress resistance of the worms (Supplementary Figure 6B), but it extended the lifespan of the wild type without affecting FMO-2 KO and FMO-2 OE, suggesting that afmd-1 requires fmo-2 to extend lifespan (Figure 6G). Knockdown of nkat-1 did not affect the lifespan or the paraquat resistance of the wild type, FMO-2 OE, and FMO-2 KO, suggesting that this gene does not

386 function in the same pathway as fmo-2 (Supplementary Figures 6C, D). Taken together, our 387 data are consistent with the hypothesis that the kynurenine pathway is a target of FMO-2. 
A

390

391

392

393

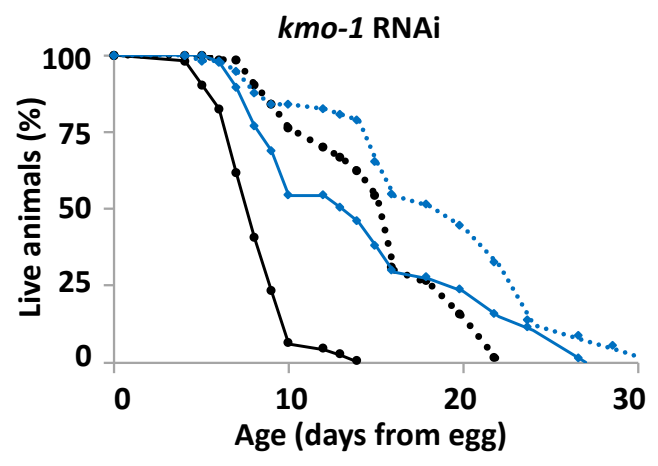

Tryptophan

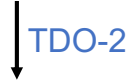

$\mathrm{N}$-formylkynurenine AMFD-1

Kynurenine $\stackrel{\text { NKATs }}{\longrightarrow}$ Kynurenic

3-Hydroxykynuerine Anthranilic acid

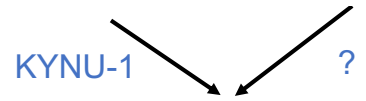

3-hyroxyanthranilic acid

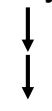

NAD+

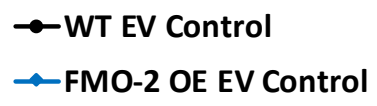

$\bullet \bullet$ WT RNAi

- . FMO-2 OE RNAi

C

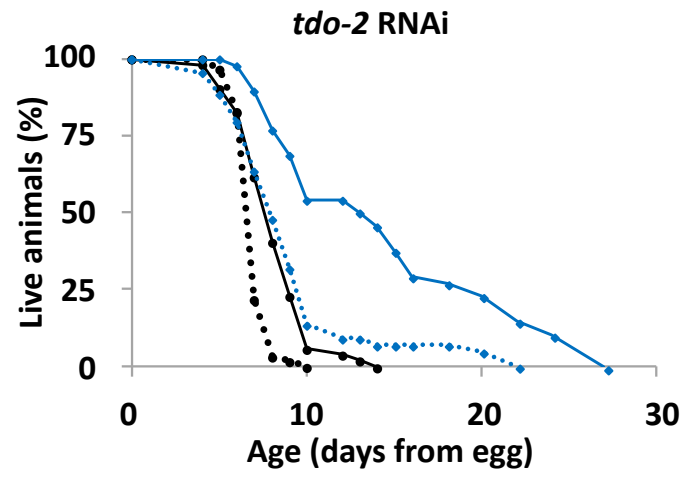

395

396

397

398

399

400

401

402

403

404

405

406 
$\rightarrow$ Wild-type EV

$\sim$ FMO-2 OE EV

$\simeq$ FMO-2 KO EV

408

D

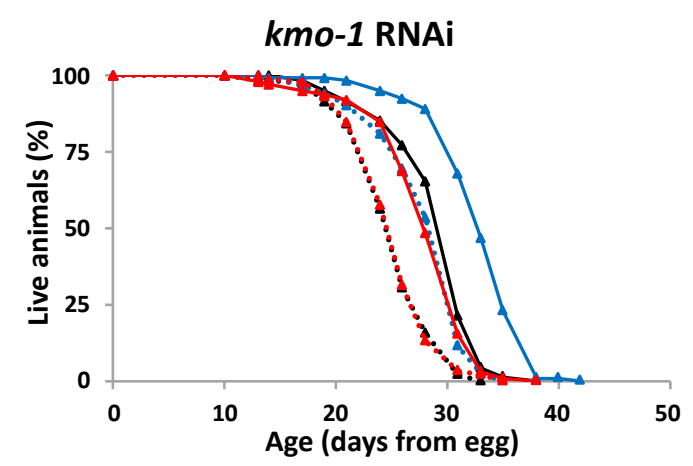

$\mathrm{F}$

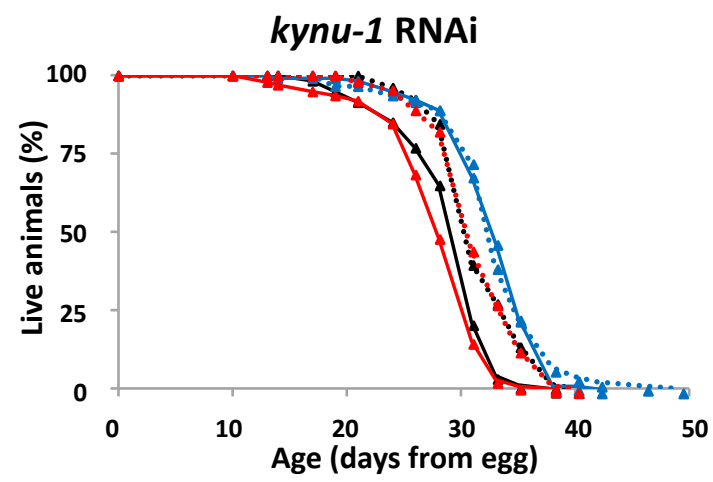

•^•Wild-type RNAi

- FMO-2 OE RNAi

- FMMO-2 KO RNAi

E

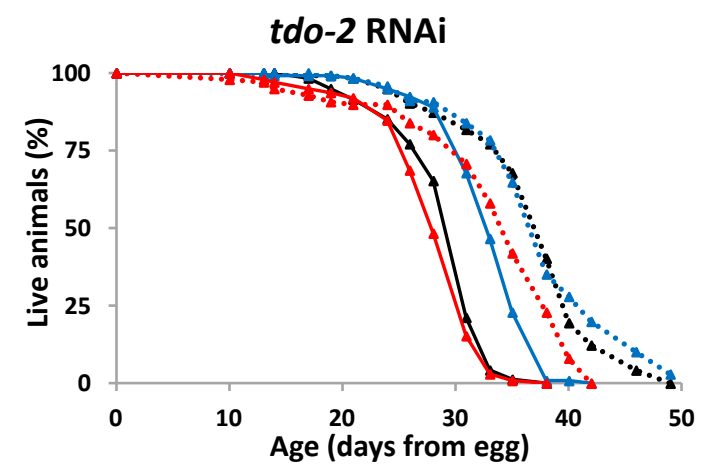

G

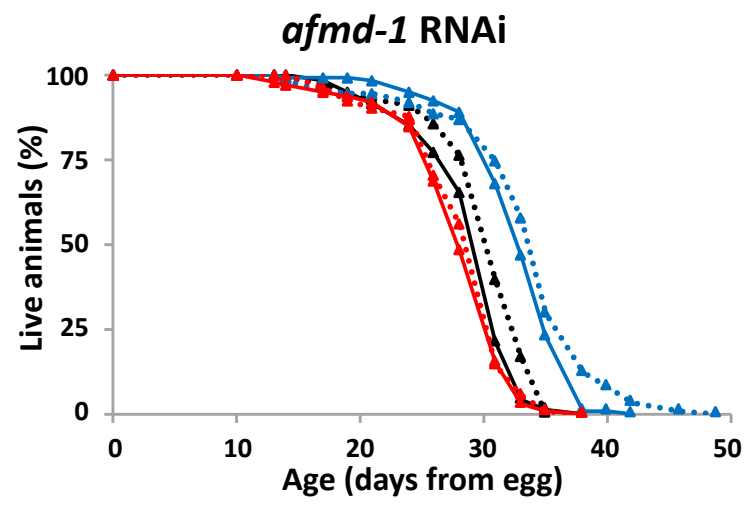

Figure 6: Fmo-2 interacts with kynurenine metabolism to regulate stress resistance and lifespan. A) Diagram of kynurenine pathway. B-C) $5 \mathrm{mM}$ paraquat stress assay (from L4 stage) comparing the survival of the wild-type and FMO-2 OE on empty vector (EV) and $B$ ) kmo-1 RNAi and C) tdo-2 RNAi, D-G) Lifespan assay comparing the survival of the wild-type, FMO-2 OE, and FMO-2 KO on EV and D) kmo-1 RNAi, E) tdo-2 RNAi, F) kynu-1 RNAi, and G) afmd-1 RNAi. Statistics in Supplementary Data 4 for stress assays and Supplementary Data 5 for lifespans. 
$\rightarrow$ WT EV Control

$\rightarrow$ FMO-2 OE EV Control

A

422

423

424

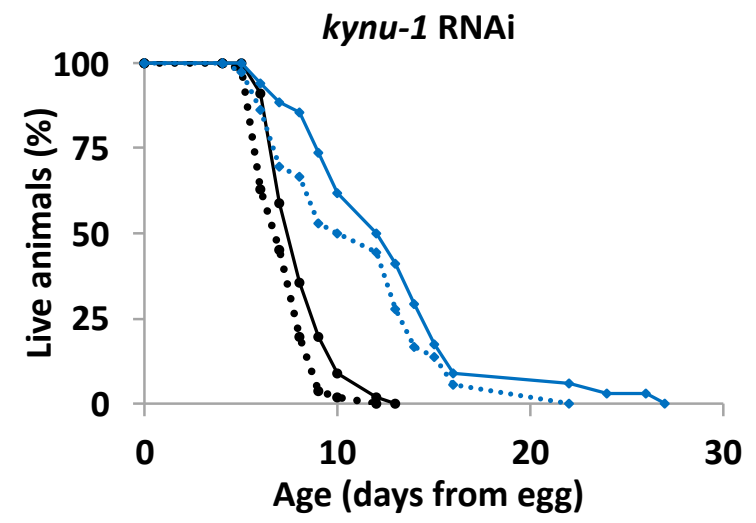

C

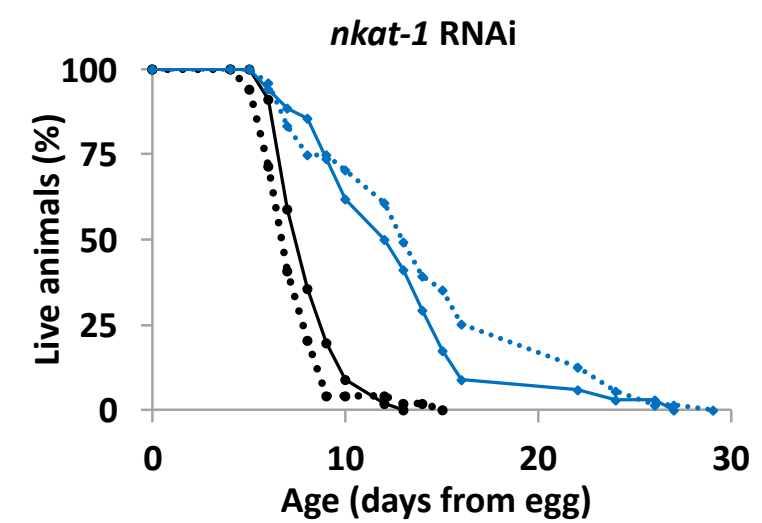

425

426

427 $\bullet \bullet \cdot$ Wild-type RNAi

$\bullet$ - FMO-2 OE RNAi

- FMO-2 KO RNAi $\leftarrow$ FMO-2 KO EV

D

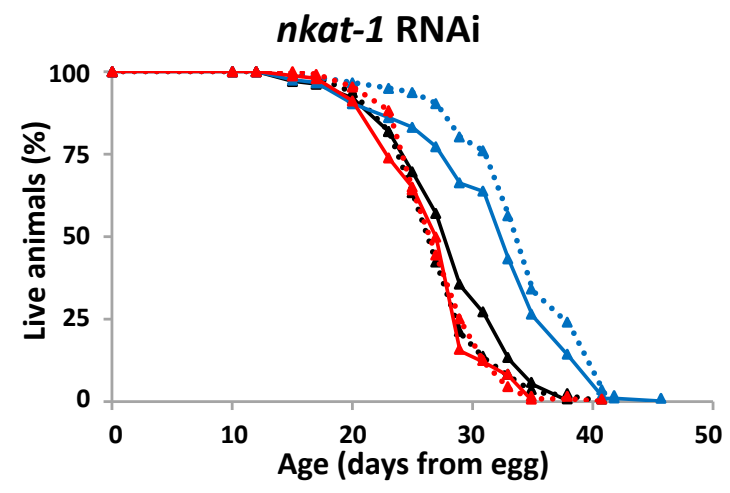

$\bullet \bullet$ WT RNAi

- . FMO-2 OE RNAi

B

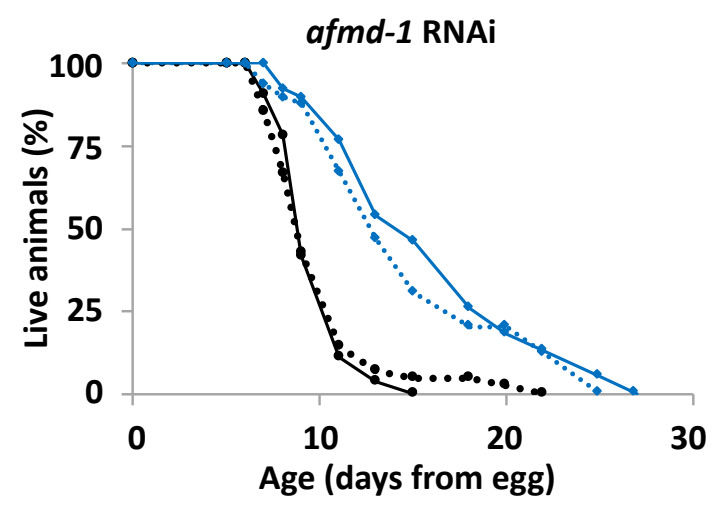

Supplementary Figure 6: Kynurenine pathway genes that do not alter worm stress resistance or lifespan.

A-C) $5 \mathrm{mM}$ paraquat stress assay

(from L4 stage) comparing the survival of the wild-type and FMO-2 OE on empty vector (EV) and $A$ ) kynu1 RNAi, B) afmd-1 RNAi, and C) nkat-1 RNAi. D) Lifespan assay comparing the survival of the wildtype, FMO-2 OE, and FMO-2 KO on EV and nkat-1 RNAi. Statistics in Supplementary Data 4 for stress assays and Supplementary Data 5 for lifespans. 


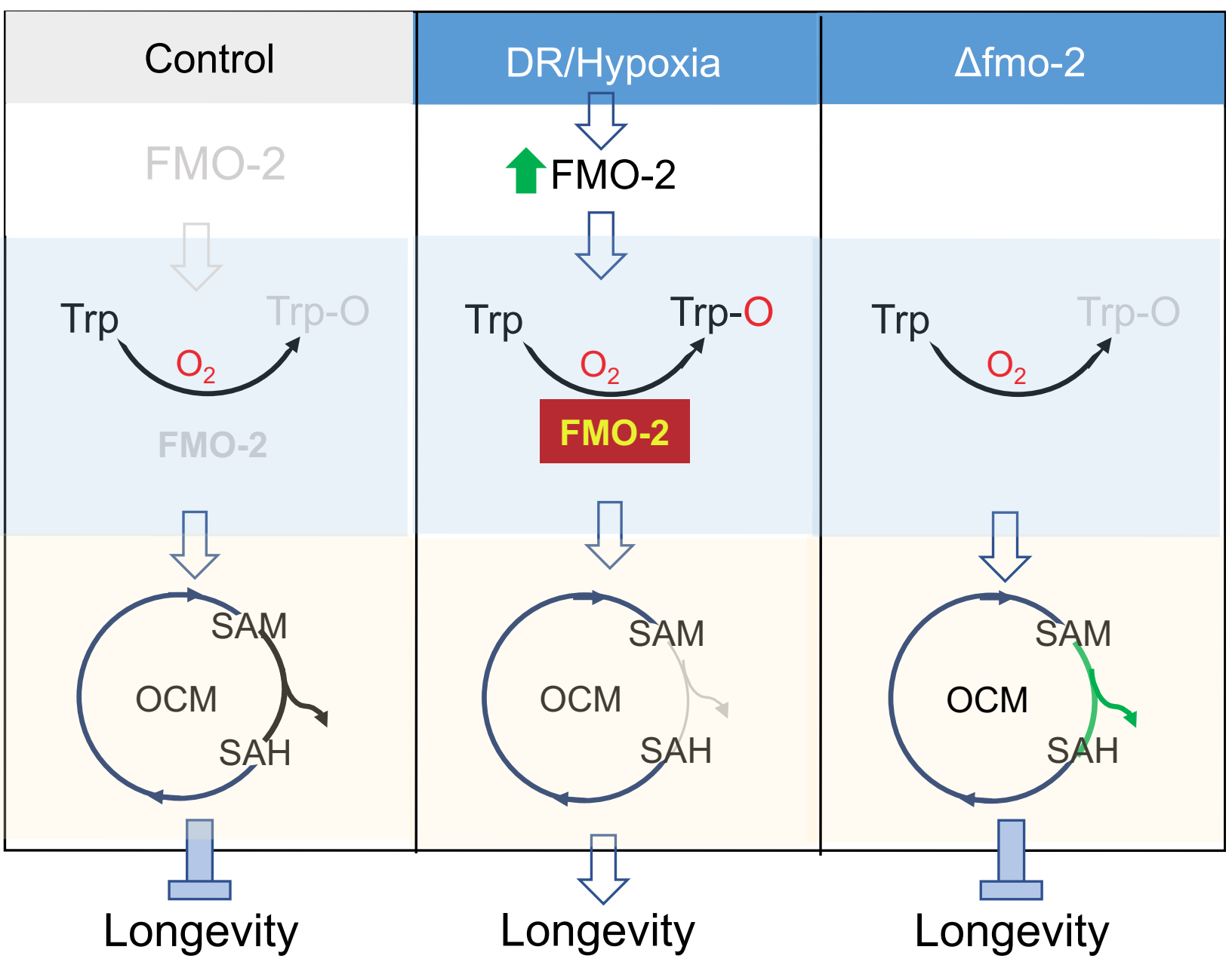

Figure 7: Proposed model. In control conditions, there is very low fmo-2 expression, leading to low levels of tryptophan metabolism/kynurenine production through FMO-2, and maintaining normal flux through one carbon metabolism and normal lifespan. When fmo-2 is induced, either genetically through overexpression or environmentally by DR or hypoxia, FMO-2 oxygenates tryptophan, leading to increased kynurenine production and decreased methylation output flux through OCM, thereby extending nematode lifespan. When fmo-2 is absent, these metabolic changes do not occur, even under hypoxia or DR, preventing an extension in lifespan. The gray line represents decreased flux and the green line represents increased flux. 
For half a century, FMOs have been primarily classified as xenobiotic enzymes. However, the mechanisms by which these enzymes affect endogenous metabolism are still not

436 well studied. Based on our data, we propose a model where overexpression of fmo-2, similar to

437 levels that we observe under hypoxia and dietary restriction, is sufficient to remodel metabolism

438 in the nematode C. elegans (Figure 7). Here, we show that Cefmo-2, a novel regulator of

439 longevity that is critical for lifespan extension and stress response under dietary restriction and

440 hypoxia, interacts with both tryptophan and one-carbon metabolism to confer longevity and

441 health benefits. We find that modulating the expression of a single oxygenating protein can have

442 a multitude of metabolic and physiological effects, similar to the activation of transcription

443 factors and kinases. Our results suggest a broader, more significant role for FMO-2, and FMOs

444 in general, than previously known. Furthermore, we establish experimental evidence of FMO

445 orthology from $C$. elegans to mammals since both CeFMO-2 and mFMO5 have similar activity

446 toward tryptophan, suggesting that through this substrate they may perform similar metabolic

447 roles in both animals.

Our resulting data are consistent with a model where the reduction of flux through the

449 methylation pathway leads to longevity benefits. By projecting gene expression data to a

450 stoichiometric model for OCM metabolism, we predict that FMO overexpression results in a

451 reduction in methylation flux. This model-based prediction based on gene expression data is

452 experimentally validated, indicating that this approach can be a powerful tool to simplify the

453 understanding of complex metabolic pathways and to study the biology of aging. Perturbation in

454 the SAM/SAH ratio by either the supplementation of metformin or a mutation in sams-1 extends

455 worm lifespan ${ }^{13,17}$. While multiple studies report that methionine restriction robustly extends

456 lifespan across species, including worms, flies, and mice ${ }^{13,43,44}$, others show that exogenous

457 supplementation of methionine is not detrimental to lifespan ${ }^{45}$. This suggests that methionine

458 utilization rather than methionine abundance is a key factor that influences the aging process. 
Although suppressing sams-1 expression phenocopies FMO-2 OE lifespan in the wild

460 type and FMO-2 KO, doing so reduces the stress resistance of the worms against paraquat.

461 This separation of lifespan and stress resistance is occasionally observed under other long-lived

462 conditions ${ }^{46}$. It is unclear if simply reducing methylation is sufficient to promote longevity

463 benefits, or if this mechanism requires suppression of specific methylation processes. It will be

464 important for future studies to determine how cells regulate different methylation fluxes under

465 sams-1 knockdown and decreased overall methylation. One potential mechanism under this

466 genetic condition could be that specific methyltransferases that are essential for survival will

467 have higher affinity to methyl groups to outcompete other nonessential or deleterious

468 methyltransferases.

We note that while our data suggest methylation as the key downstream effector of

470 FMO-2, we have not excluded the possibility that the transsulfuration pathway may also be

471 involved in this mechanism. The transsulfuration pathway is reported to be a necessary and

472 sufficient component of DR-mediated lifespan extension in flies ${ }^{16}$. Similarly, knocking down cth-

4732 , a gene involved in this pathway, abrogates the lifespan extension phenotype in FMO-2 OE

474 (Figure 3B). It will be interesting to determine the mechanistic relationship between the

475 transsulfuration and transmethylation pathways in regulating longevity.

476 Our data also support an interaction between fmo-2 and tryptophan metabolism to

477 influence longevity. These findings are particularly interesting because we identify a putative

478 endogenous metabolic pathway of FMOs in relation to the aging process. Based on cell line

479 metabolomics, enzyme kinetics, and HPLC data, there are at least two plausible mechanisms

480 for how oxygenation of tryptophan by FMO-2 can lead to the synthesis of $\mathrm{N}$-formylkynurenine.

481 First, FMOs across taxa are known to dimerize and form higher order oligomers ${ }^{47,48}$. Therefore,

482 it is possible that FMO-2 dimerizes and dioxygenates tryptophan forming $\mathrm{N}$-formyl-kynurenine,

483 which is then converted to kynurenine by formamidase. Second, the same process could be

484 involved in subsequent oxygenation by FMO-2 monomers, but it is unknown how stable a 
monooxygenated form of tryptophan would be within the cell, making the first mechanism more likely. To our knowledge this is the first example of the dioxygenation of a substrate that could

\section{7} potentially require dimerized FMOs. The mechanism of this reaction and its potential requirement of dimerized FMOs will be a target of future research. Furthermore, the dioxygenation of tryptophan by FMOs is especially interesting considering only dioxygenases, such as tdo-2, ido-1, and ido-2, have been shown to mediate the conversion of tryptophan to $\mathrm{N}$ formylkynurenine ${ }^{19}$. Regardless, our data implicate tryptophan as a bona fide in vitro and likely in vivo substrate of animal FMOs either through dioxygenating or monooxygenating mechanisms. Although we tested multiple conventional and unconventional FMO substrates, such as methimazole ${ }^{47-49}$ and 2-heptanone ${ }^{50}$ (Supplementary Data 9), respectively, much work remains to fully establish the general FMO-2 substrate profile and how it compares to those of mammalian FMOs beyond the common tryptophan substrate of CeFMO-2 and mFMO5.

This is the first report showing the conservation of enzymatic activity toward a potential posited as the ortholog of FMO-2 and the other $C$. elegans FMOs due to it being the most ancestral mammalian FMO, experimental evidence demonstrating this has been lacking. The alignment of $C$. elegans FMO-2 with mFMO5 illustrates the highly conserved putative catalytic residues amongst the FMOs (Supplementary Figure 5). This conserved activity is demonstrated further here in the similar activities of CeFMO-2 and mFMO5 toward tryptophan as well as the production of $\mathrm{N}$-formylkynurenine from tryptophan. As we show here

505 metabolomics analysis reveals that multiple human cell lines with overexpression of mFMOs

506 have less tryptophan ${ }^{10}$. Altogether, our previous data and the data presented here further 507 suggest that not only is tryptophan a bona fide endogenous target of $\mathrm{C}$. elegans FMOs but 508 potentially of mFMOs as well. metabolism directly or indirectly modulates the metabolite profile of OCM, altering flux patterns 
511 that are consistent with our computational model predictions and subsequent genetic analyses.

512 Further investigation is needed to understand the fmo-2-mediated connection between OCM

513 and tryptophan in regulating lifespan. Based on the knowledge we gained from this study and

514 previous work, we propose the following possibilities: 1) Oxygenation of tryptophan by FMO-2

515 alters OCM flux by increasing formate levels as a potential direct link between tryptophan

516 metabolism and OCM. Formate is a single carbon-containing molecule that can enter the folate

517 cycle as a carbon source ${ }^{42}$. Formate is generated as a byproduct when kynurenine is

518 synthesized from $\mathrm{N}$-formylkynurenine by formamidase $^{42}$. It is possible that increased of formate

519 levels can confer stress resistance and longevity benefits under metabolically stressful

520 conditions, such as DR or hypoxia. 2) FMO-2 interacts with the mechanistic target of rapamycin

521 (mTOR). Dietary restriction leads to inhibition of mTOR signaling, which is a central regulator of

522 lifespan and aging ${ }^{51}$. Interestingly, both DR- and rapamycin-mediated mTOR inhibition induce

523 the expression of FMOs. A recent study shows that diaminodiphenyl sulfone (DDS) induces the

524 expression of fmo-2 and extends lifespan, but it does not further extend lifespan in combination

525 with rapamycin ${ }^{37}$. This finding is consistent with the hypothesis that fmo-2 interacts with mTOR

526 inhibition to extend lifespan. We show that fmo-2 interacts with SAM and tryptophan

527 metabolism, both of which are known to alter mTOR activity ${ }^{52-54}$. Thus, examination into the role

528 of mTOR in fmo-2-mediated lifespan extension is warranted. 3) FMO-2 modulates tryptophan

529 metabolism and OCM in an independent manner. There is a possibility that there is no

530 connection between OCM and tryptophan, and FMO-2 targets both pathways independently.

531 Taken together, our study expands the role of FMO-2 from a xenobiotic enzyme to a

532 metabolic regulator of longevity that has global effects on the metabolome in worms. In

533 particular, the identification of OCM as a target of FMO-2 has implications outside the aging

534 field, considering that OCM remodeling has been studied under the context of cancer biology for

535 more than 70 years $^{55}$. Furthermore, through the identification of tryptophan as a putative

536 substrate for both CeFMO-2 and mFMO5, this study highlights the conserved importance of 
537 FMOs in multiple contexts, including aging and many diseases where OCM and/or the

538 kynurenine pathway play a role. These findings illustrate the potential for therapeutic targets of

539 these proteins for treating age-related diseases and/or increasing longevity and healthspan.

540 This exciting translational potential for the conserved roles of FMOs will be a target for future

541 research. 


\section{Materials and Methods}

\section{Strains and Growth Conditions}

Standard C. elegans cultivation procedures were used as previously described ${ }^{4,56}$. N2 wild type, KAE9 ((eft-3p::fmo-2 + h2b::gfp + Cbr-unc-119(+)), and VC1668 (fmo-2(ok2147))

547 strains were maintained on solid nematode growth media (NGM) using E. coli OP50 throughout 548 life except where RNAi (E. coli HT115) were used. All experiments were conducted at $20^{\circ} \mathrm{C}$.

$\underline{\text { Metabolomics }}$

OP50 bacteria were treated with $0.5 \%(\mathrm{v} / \mathrm{v})$ paraformaldehyde as previously described ${ }^{56}$

552 and seeded onto $100 \mathrm{~mm}$ NGM plates. Approximately 500 eggs were put on these plates and

553 grown until they reached late L4 larval stage. The worms were washed off the plates with 10

$554 \mathrm{~mL}$ of M9 buffer and were collected in $15 \mathrm{~mL}$ conical tubes. The worms were pelleted using a

555 clinical centrifuge for 1 minute at $150 \times \mathrm{g}$ and the supernatant was vacuum aspirated. The worms were washed once with $10 \mathrm{~mL}$ of M9 buffer and then with $10 \mathrm{~mL}$ of $150 \mathrm{mM}$ ammonium

557 acetate to remove phosphates from M9, each time being centrifuged and the supernatant being 558 aspirated. After these washing steps, the pellets were flash frozen in liquid nitrogen. Metabolites were extracted from pellets by addition of $500 \mu \mathrm{L}$ of ice-cold 9:1 methanol: chloroform, followed immediately by probe sonication for 30 seconds with a Branson 450

561 Sonicator. The resulting homogenates were kept on ice for 5 minutes and were then centrifuged

562 for 10 minutes at $4000 \times \mathrm{g}$ at $4^{\circ} \mathrm{C}$. Supernatant was then transferred to autosampler vials for

563 analysis. Hydrophilic interaction liquid chromatography-electrospray ionization mass

564 spectrometry (HILIC-LC-ESI-MS) analysis was performed in negative ion mode using an Agilent 5651200 LC system coupled to an Agilent 6220 time-of-flight mass spectrometer. Chromatography 566 was performed as previously described ${ }^{57,58}$, with the exception that the Phenomenex Luna NH2 567 column used had dimensions of $150 \mathrm{~mm} \times 1.0 \mathrm{~mm} \mathrm{ID}$, the flow rate was $0.07 \mathrm{~mL} / \mathrm{min}$, and the 
568 injection volume was $10 \mu \mathrm{L}$. Untargeted peak detection and alignment was performed using $569 \mathrm{XCMS}^{59}$.

$570 \quad$ The resulting metabolomics data were analyzed using Metaboanalyst 4.0

571 (http://metaboanalyst.ca). Within Metaboanalyst, the data were median normalized, adjusted

572 using auto scaling, and were then subjected to principal component analysis using default

573 parameters. Pathway analysis was performed using Metaboanalyst's functional analysis

574 module. P-values and t-scores of each MS peak data were calculated between the wild type

575 and FMO-2 OE (Supplementary Data 2). Mass tolerance was set to 10 parts per million (ppm)

576 and mummichog algorithm p-value cutoff was set to 0.05. Default parameters were used for

577 other settings and the analysis was done using the C. elegans pathway library.

578 Targeted metabolomics analysis used the same LC-MS parameters as untargeted, but

579 data analysis was performed using Agilent MassHunter Quantitative Analysis software.

580 Metabolite identification was performed by matching accurate mass and retention time with

581 authentic standards analyzed by the same method. Data were normalized to the median and log

582 transformed. Statistical analysis for targeted metabolomics data was done using Metaboanalyst.

Stress resistance assay

Paraquat (Methyl viologen dichloride hydrate from Sigma-Aldrich) was used to induce oxidative stress. Worms were synchronized from eggs on RNAi plates seeded with $E$.

587 coli HT115 strain expressing dsRNAi for a particular gene and at L4 stage 40 worms were

588 transferred on RNAi-FUDR plates containing $5 \mathrm{mM}$ paraquat. A minimum of two plates per

589 strain per condition were used per replicate experiment. Worms were then scored every day

590 and considered dead when they did not move in response to prodding under a dissection

591 microscope. Worms that crawled off the plate were not considered, but ruptured worms were

592 noted and considered as previously described ${ }^{4}$. 
Gravid adults were placed on NGM plates containing $1 \mathrm{mM} \beta$-D-isothiogalactopyranoside (IPTG), $25 \mu \mathrm{g} / \mathrm{ml}$ carbenicillin, and the corresponding RNAi clone from the Vidal or Ahringer

597 RNAi library. After 3 hours, the adults were removed, and the eggs were allowed to develop at $59820^{\circ} \mathrm{C}$ until they reached late L4/young adult stage. From here, 40 to 90 worms were placed on 599 each RNAi plate and transferred to fresh RNAi + FUDR plates on day 1, day 2, day 4, and day 6

600 of adulthood. A minimum of two plates per strain per condition were used per replicate

601 experiment. Experimental animals were scored every 2-3 days and considered dead when they

602 did not move in response to prodding under a dissection microscope. Worms that crawled off

603 the plate were not considered, but ruptured worms were considered as previously described ${ }^{4}$.

Computational Modeling

The computer model was generated by building a stoichiometric matrix $S$ (10 reactants

by 13 reactions), accounting for all reactions shown in Fig 4A. A steady-state approximation was used, as shown in Eq 1. In Eq. 1, $\mathbf{S}$ is the stoichiometric matrix and $\mathbf{J}$ is a vector of fluxes for each of the reactions.

Eq. 1

To obtain a biologically relevant solution, we projected the expression data of genes

615 involved in the reactions used in the model to the nullspace of $S$ by solving for Eq. 2. Single

616 genes were used as representative genes for each reaction to simplify the model. Gene

617 expressions related to input fluxes were assumed to be one for all strains. Reactions used in the

618 model and the relevant gene expression data are shown in Supplementary Data 7. In Eq. 2, M

619 is the nullspace of $\mathbf{S}, \mathbf{b}$ is the vector of relative gene expression data from the wild type, FMO-2 
620 OE or FMO-2 KO that have been normalized to the wild type, and $\mathbf{x}$ is a vector such that Sx is

621 the projection of $\mathbf{b}$ onto the column space of $M$, which gives us the vector of reaction fluxes, $\mathbf{J}$,

622 within the nullspace of S. To account for data variability, expression level with greater than $0.5 x$

623 or less than $1.5 x$ fold changes were assumed to be equal to the wild type control. Eq. 2 was

624 solved using the Isqminnorm function in MATLAB 2018a. The Isqminnorm function returns the

625 minimum norm least-squares solution to $\mathbf{M x}=\mathbf{b}$ by minimizing both the norm of $\mathbf{M}{ }^{*} \mathbf{x}-\mathbf{b}$ and

626 the norm of $\mathbf{x}$.

627

628 Eq. 2

$M \cdot \mathbf{X}=\mathbf{b}$

630

The inner product of the resulting vector $\mathbf{x}$ and the nullspace matrix $M$ was obtained to

632 calculate the reaction flux predictions resulting from the gene expression projection as shown in

633 Eq. 3. The calculated $\mathbf{J}$ for FMO-2 OE and FMO-2 KO were normalized to that of the wild type

634 to obtain the relative fluxes.

$$
\mathrm{M} \cdot \mathbf{x}=\mathbf{J}
$$

Quantitative PCR

RNA was isolated from day 1 adult worms following three rounds of freeze-thaw in liquid

641 nitrogen using Invitrogen's Trizol extraction method and $1 \mu \mathrm{g}$ of RNA was reverse transcribed to

642 cDNA using SuperScript ${ }^{\mathrm{TM}}$ II Reverse Transcriptase (Invitrogen). Gene expression levels were

643 measured using $1 \mu \mathrm{g}$ of cDNA and SYBR ${ }^{\mathrm{TM}}$ Green PCR Mastermix (Applied Biosystems) and

644 primers at $10 \mu \mathrm{M}$ concentration. mRNA levels were normalized using previously published 
645 housekeeping gene controls, tba-1 and $p m p-3^{60}$. List of primers used are in Supplementary

646 Data 11.

648 Enzyme Kinetic Assays

649 Oxygenation activity of FMO-2 and mFMO5 was characterized using the method

650 previously described ${ }^{61}$. Briefly, oxygenation of substrates was determined by

651 spectrophotometrically following the consumption of NADPH at $340 \mathrm{~nm}$ using the molar

652 extinction coefficient $6.22 \mathrm{mM}^{-1} \mathrm{~cm}^{-1}$. Components of the assay buffer included $25 \mathrm{mM}$ sodium

653 phosphate buffer ( $\mathrm{pH} 8.5), 0.5 \mathrm{mM}$ diethylenetriaminepentaacetic acid (DETAPAC), $0.5 \mathrm{mM}$

$654 \mathrm{NADPH}$, and $0.04 \mu \mathrm{M}$ FMO-2 (0.4 $\mu \mathrm{M}$ FMO5) with excess FAD. The final substrate

655 concentrations for tryptophan were 100, 250, 500, $750 \mu \mathrm{M}$ and 1, 2.5, 5, 7.5, and $10 \mathrm{mM}$. The

656 final substrate concentrations for $\mathrm{MMI}$ were 100,300 , and $600 \mu \mathrm{M}$ and $1,3,5,7,10$, and 30

$657 \mathrm{mM}$. To determine the rate of oxidation of NADPH by FMO, NADPH concentrations of 10, 30,

$658100,300,500$, and $700 \mu \mathrm{M}$ and 1 and $1.5 \mathrm{mM}$ were used. Experiments were conducted at $30^{\circ} \mathrm{C}$

659 while shaking. Kinetic parameters (i.e $k_{c a t}$ and $K_{m}$ ) were determined by fitting plots of the rate of

660 turnover vs the substrate concentration to the Michaelis-Menton equation using GraphPad

661 Prism (version 9.1.0; GraphPad Software Inc., San Diego, CA.). Purified FMO-2 protein was

662 purchased from GenScript. Purified FMO5 protein, NADPH, FAD, MMI, L-tryptophan, and all

663 other substrates were purchased from Sigma Aldrich (St. Louis, MO). DETAPAC and sodium

664 phosphate buffer were purchased from Fisher (Waltham, MA).

666 In vitro studies LC-MS

667 Analysis of samples from in vitro studies with purified FMO2 and FMO5 protein was performed

668 using LC-MS with untargeted feature detection. Samples contained 100, 250, or $500 \mu \mathrm{M}$

669 tryptophan in the same conditions as the enzymatic assays with either FMO-2 or FMO5

670 proteins. $100 \mu \mathrm{L}$ of conditioned media were vortexed with $400 \mu \mathrm{L}$ of $1: 1: 1$ 
671 methanol:acetonitrile:acetone to precipitate protein. The extract was centrifuged for 10 minutes

672 at $16,000 \times g$ and $200 \mu \mathrm{L}$ of supernatant were transferred to a clean autosampler vial with insert

673 and dried under a stream of nitrogen gas. The dried extract was reconstituted in $50 \mu \mathrm{L}$ of $85 / 15$

674 acetonitrile/water and analyzed by HILIC-TOF-MS on an Agilent 1290 Infinity II / Agilent 6545

675 QTOF. Chromatography was performed on a Waters BEH Amide column $(2.1 \mathrm{~mm} \mathrm{ID} \mathrm{x} 10 \mathrm{~cm}$,

$6761.7 \mu \mathrm{m}$ particle diameter) with mobile phase prepared as described previously ${ }^{62}$ except that

677 mobile phase A contained $5 \%$ acetonitrile. The flow rate was $0.3 \mathrm{~mL} / \mathrm{min}$, the column

678 temperature $55^{\circ} \mathrm{C}$, and the gradient was as follows: $0-0.70 \min 100 \% \mathrm{~B}, 0.7-6.7 \min 100-85 \% \mathrm{~B}$,

$679 \quad 6.7-8.7 \min 85 \% \mathrm{~B}, 8.7-16 \min 85-28 \% \mathrm{~B}, 16-16.7 \min 28 \% \mathrm{~B}, 16.7-16.828-0 \% \mathrm{~B}$. Total run time

680 was $22 \mathrm{~min}$. Ion polarity was positive, gas temp was $320^{\circ} \mathrm{C}$, drying gas was $8 \mathrm{~L} / \mathrm{min}$, nebulizer

681 was $35 \mathrm{psi}$, sheath gas temp and flow were $350^{\circ} \mathrm{C}$ and $11 \mathrm{~L} / \mathrm{min}$, capillary voltage $3500 \mathrm{~V}$. The

682 instrument was operated in full scan mode at 2 spectra/sec and a mass range of 50-1200 Da.

683 Feature detection and alignment was performed using XCMS. Potential reaction products were

684 detected by computationally examining the data for features present in each sample set.

685 Identification of potential reaction products was performed using MS/MS data acquired from a 686 pooled sample.

688 Statistical analyses

689 Log-rank test was used to derive p-value for lifespan and paraquat survival assays using $690 p<0.0001$ cut-off threshold compared to EV controls. Paired-test was used to derive $p$-values

691 for targeted metabolomics data using $p<0.05$ cut-off threshold compared to the wild type. One-

692 way ANOVA followed by Tukey's post hoc test was used to derive p-values for SAM/SAH ratio 693 using $p<0.05$ cut-off threshold. Paired t-test was used to derive $p$-values for comparing the 694 metabolomics data of HepG2 pDEST control and FMO2, FMO4, and FMO5 OE cell lines using $695 p<0.05$ cut-off threshold. 


\section{References}

698 1. Rossner R, Kaeberlein M, Leiser SF. Flavin-containing monooxygenases in aging and disease: Emerging roles for ancient enzymes. J Biol Chem. 2017. doi:10.1074/jbc.R117.779678

2. Ziegler DM. An overview of the mechanism, substrate specificities, and structure of FMOs. In: Drug Metabolism Reviews. ; 2002. doi:10.1081/DMR-120005650

3. Krueger SK, Williams DE. Mammalian flavin-containing monooxygenases:

705 Structure/function, genetic polymorphisms and role in drug metabolism. Pharmacol Ther.

4. Leiser SF, Miller H, Rossner R, et al. Cell nonautonomous activation of flavin-containing monooxygenase promotes longevity and health span. Science (80- ). 2015.

708 doi:10.1126/science.aac9257

709

5. Steinbaugh MJ, Sun LY, Bartke A, Miller RA. Activation of genes involved in xenobiotic metabolism is a shared signature of mouse models with extended lifespan. Am J Physiol Metab. 2012. doi:10.1152/ajpendo.00110.2012

6. Swindell WR. Genes and gene expression modules associated with caloric restriction and aging in the laboratory mouse. BMC Genomics. 2009. doi:10.1186/1471-2164-10-585

714 7. Bennett CF, Kwon JJ, Chen C, et al. Transaldolase inhibition impairs mitochondrial respiration and induces a starvation-like longevity response in Caenorhabditis elegans.

717 8. Veeravalli S, Omar BA, Houseman L, et al. The phenotype of a flavin-containing monooyxgenase knockout mouse implicates the drug-metabolizing enzyme FMO1 as a novel regulator of energy balance. Biochem Pharmacol. 2014. doi:10.1016/j.bcp.2014.04.007

721 9. Scott F, Gonzalez Malagon SG, O'Brien BA, et al. Identification of flavin-containing 
monooxygenase 5 (FMO5) as a regulator of glucose homeostasis and a potential sensor of gut bacteria. Drug Metab Dispos. 2017. doi:10.1124/dmd.117.076612

10. Huang S, Howington MB, Dobry CJ, Evans CR, Leiser SF. Flavin-Containing Monooxygenases Are Conserved Regulators of Stress Resistance and Metabolism. Front Cell Dev Biol. 2021;9. doi:10.3389/fcell.2021.630188

727 11. Gao AW, Smith RL, van Weeghel M, Kamble R, Janssens GE, Houtkooper RH. Identification of key pathways and metabolic fingerprints of longevity in C. elegans. Exp

12. Menendez JA, Joven J. One-carbon metabolism: An aging-cancer crossroad for the gerosuppressant metformin. Aging (Albany NY). 2012.

732 13. Cabreiro F, Au C, Leung KY, et al. Metformin retards aging in C. elegans by altering microbial folate and methionine metabolism. Cell. 2013. doi:10.1016/j.cell.2013.02.035

734 14. Annibal A, George Tharyan R, Fides Schonewolff M, et al. Regulation of the one carbon folate cycle as a shared metabolic signature of longevity. doi:10.1038/s41467-021-23856-

737 15. Locasale JW. Serine, glycine and one-carbon units: Cancer metabolism in full circle. Nat Rev Cancer. 2013. doi:10.1038/nrc3557

16. Kabil H, Kabil O, Banerjee R, Harshman LG, Pletcher SD. Increased transsulfuration mediates longevity and dietary restriction in Drosophila. Proc Natl Acad Sci. 2011.

17. Hansen M, Hsu A-L, Dillin A, Kenyon C. New Genes Tied to Endocrine, Metabolic, and Dietary Regulation of Lifespan from a Caenorhabditis elegans Genomic RNAi Screen. Kim S, ed. PLoS Genet. 2005;1(1):e17. doi:10.1371/journal.pgen.0010017

18. Oxenkrug GF. The extended life span of Drosophila melanogaster eye-color (white and vermilion) mutants with impaired formation of kynurenine. J Neural Transm. 2010. doi:10.1007/s00702-009-0341-7 
19. Van Der Goot AT, Zhu W, Vázquez-Manrique RP, et al. Delaying aging and the agingassociated decline in protein homeostasis by inhibition of tryptophan degradation. Proc Natl Acad Sci U S A. 2012. doi:10.1073/pnas.1203083109

20. Sutphin GL, Backer G, Sheehan S, et al. Caenorhabditis elegans orthologs of human genes differentially expressed with age are enriched for determinants of longevity. Aging Cell. 2017. doi:10.1111/acel.12595

21. Badawy AAB. Kynurenine pathway of tryptophan metabolism: Regulatory and functional aspects. Int J Tryptophan Res. 2017;10(1). doi:10.1177/1178646917691938

22. Liu YJ, Janssens GE, Mclntyre RL, et al. Glycine promotes longevity in caenorhabditis elegans in a methionine cycle-dependent fashion. PLoS Genet. 2019. doi:10.1371/journal.pgen.1007633

23. Depuydt G, Xie F, Petyuk VA, et al. LC-MS proteomics analysis of the insulin/IGF-1deficient caenorhabditis elegans daf-2(e1370) mutant reveals extensive restructuring of intermediary metabolism. J Proteome Res. 2014. doi:10.1021/pr401081b

24. Johnson TE, Cypser J, De Castro E, et al. Gerontogenes mediate health and longevity in nematodes through increasing resistance to environmental toxins and stressors. In: Experimental Gerontology. ; 2000. doi:10.1016/S0531-5565(00)00138-8

25. Finkel T, Holbrook NJ. Oxidants, oxidative stress and the biology of ageing. Nature. 2000. doi:10.1038/35041687

26. Johnson TE, Henderson S, Murakami S, et al. Longevity genes in the nematode Caenorhabditis elegans also mediate increased resistance to stress and prevent disease. J Inherit Metab Dis. 2002. doi:10.1023/A:1015677828407

27. Miller RA. Cell stress and aging: New emphasis on multiplex resistance mechanisms. In: Journals of Gerontology - Series A Biological Sciences and Medical Sciences. ; 2009. doi:10.1093/gerona/gln072

773 28. Kruempel JCP, Miller HA, Schaller ML, et al. Hypoxic response regulators RHY-1 and 
EGL-9/PHD promote longevity through a VHL-1-independent transcriptional response. GeroScience. 2020;42(6):1621-1633. doi:10.1007/s11357-020-00194-0

776

29. Lee HJ, Noormohammadi A, Koyuncu S, et al. Prostaglandin signals from adult germline stem cells delay somatic ageing of Caenorhabditis elegans. Nat Metab. 2019;1(8):790-

778 810. doi:10.1038/s42255-019-0097-9

779 30. Hine C, Harputlugil E, Zhang Y, et al. Endogenous hydrogen sulfide production is essential for dietary restriction benefits. Cell. 2015;160(1-2):132-144.

781 doi:10.1016/j.cell.2014.11.048

782 31. Wei $\mathrm{Y}$, Kenyon $\mathrm{C}$. Roles for ROS and hydrogen sulfide in the longevity response to germline loss in Caenorhabditis elegans. Proc Natl Acad Sci U S A. 2016;113(20):E2832E2841. doi:10.1073/pnas.1524727113

32. Ding W, Smulan LJ, Hou NS, Taubert S, Watts JL, Walker AK. s-Adenosylmethionine Levels Govern Innate Immunity through Distinct Methylation-Dependent Pathways. Cell Metab. 2015;22:633-645. doi:10.1016/j.cmet.2015.07.013

33. Obata F, Miura M. Enhancing S-adenosyl-methionine catabolism extends Drosophila lifespan. Nat Commun. 2015;6(1):1-9. doi:10.1038/ncomms9332

34. Orth JD, Thiele I, Palsson BO. What is flux balance analysis? Nat Biotechnol. 2010. doi:10.1038/nbt.1614

792 35. Singh S, Samal A, Giri V, Krishna S, Raghuram N, Jain S. Flux-based classification of reactions reveals a functional bow-tie organization of complex metabolic networks. Phys Rev E - Stat Nonlinear, Soft Matter Phys. 2013. doi:10.1103/PhysRevE.87.052708

36. Walker AK, Jacobs RL, Watts JL, et al. A conserved SREBP-1/phosphatidylcholine feedback circuit regulates lipogenesis in metazoans. Cell. 2011. doi:10.1016/j.cell.2011.09.045

37. Choi H, Cho SC, Ha YW, et al. DDS promotes longevity through a microbiome-mediated starvation signal. Trans/ Med Aging. 2019;3:64-69. doi:10.1016/j.tma.2019.07.001 
800

801

802

803

804

805

806

807

808

809

810

811

812

813

814

815

816

817

818

819

820

821

822

823

824

825

38. Yi P, Melnyk S, Pogribna M, Pogribny IP, Hine RJ, James SJ. Increase in plasma homocysteine associated with parallel increases in plasma S-adenosylhomocysteine and lymphocyte DNA hypomethylation. J Biol Chem. 2000;275(38):29318-29323.

doi:10.1074/jbc.M002725200

39. Nicoll CR, Bailleul G, Fiorentini F, Mascotti ML, Fraaije MW, Mattevi A. Ancestralsequence reconstruction unveils the structural basis of function in mammalian FMOs. Nat Struct Mol Biol. 2020;27(1):14-24. doi:10.1038/s41594-019-0347-2

40. Sievers F, Wilm A, Dineen D, et al. Fast, scalable generation of high-quality protein multiple sequence alignments using Clustal Omega. Mol Syst Biol. 2011;7. doi:10.1038/msb.2011.75

41. Robert X, Gouet P. Deciphering key features in protein structures with the new ENDscript server. Nucleic Acids Res. 2014;42(W1). doi:10.1093/nar/gku316

42. Brosnan ME, Brosnan JT. Formate: The Neglected Member of One-Carbon Metabolism. 2016. doi:10.1146/annurev-nutr-071715-050738

43. Sun L, Sadighi Akha AA, Miller RA, Harper JM. Life-span extension in mice by preweaning food restriction and by methionine restriction in middle age. Journals Gerontol - Ser A Biol Sci Med Sci. 2009. doi:10.1093/gerona/glp051

44. Lee BC, Kaya A, Ma S, et al. Methionine restriction extends lifespan of Drosophila melanogaster under conditions of low amino-acid status. Nat Commun. 2014. doi:10.1038/ncomms4592

45. Parkhitko AA, Binari R, Zhang N, Asara JM, Demontis F, Perrimon N. Tissue-specific down-regulation of S-adenosyl-homocysteine via suppression of dAhcyL1/dAhcyL2 extends health span and life span in Drosophila. Genes Dev. 2016. doi:10.1101/gad.282277.116

46. Dues DJ, Andrews EK, Senchuk MM, Van Raamsdonk JM. Resistance to Stress Can Be Experimentally Dissociated From Longevity. Journals Gerontol Ser A. 2019;74(8):1206- 
1214. doi:10.1093/gerona/gly213

827 47. Reddy RR, Ralph EC, Motika MS, Zhang J, Cashman JR. Characterization of human 828 flavin-containing monooxygenase (FMO) 3 and FMO5 expressed as maltose-binding protein fusions. Drug Metab Dispos. 2010. doi:10.1124/dmd.110.033639

48. Lončar N, Fiorentini F, Bailleul G, et al. Characterization of a thermostable flavincontaining monooxygenase from Nitrincola lacisaponensis (NiFMO). Appl Microbiol Biotechnol. 2019;103(4):1755-1764. doi:10.1007/s00253-018-09579-w

49. Chen Y, Patel NA, Crombie A, Scrivens JH, Murrell JC. Bacterial flavin-containing monooxygenase is trimethylamine monooxygenase. Proc Natl Acad Sci. 2011. doi:10.1073/pnas.1112928108

50. Fiorentini F, Geier M, Binda C, et al. Biocatalytic Characterization of Human FMO5: Unearthing Baeyer-Villiger Reactions in Humans. ACS Chem Biol. 2016;11(4):10391048. doi:10.1021/acschembio.5b01016

51. Kapahi P, Kaeberlein M, Hansen M. Dietary restriction and lifespan: Lessons from invertebrate models. Ageing Res Rev. 2017;39:3-14. doi:10.1016/j.arr.2016.12.005

52. Gu X, Orozco JM, Saxton RA, et al. SAMTOR is an S-adenosylmethionine sensor for the mTORC1 pathway. Science (80- ). 2017;358(6364):813-818. doi:10.1126/science.aao3265

53. Metz R, Rust S, DuHadaway JB, et al. IDO inhibits a tryptophan sufficiency signal that stimulates mTOR: A novel IDO effector pathway targeted by D-1-methyl-tryptophan. Oncoimmunology. 2012;1(9):1460-1468. doi:10.4161/onci.21716

54. Wang H, Ji Y, Wu G, et al. L-Tryptophan activates mammalian target of rapamycin and enhances expression of tight junction proteins in intestinal porcine epithelial cells. J Nutr. 2015;145(6):1156-1162. doi:10.3945/jn.114.209817

55. Newman AC, Maddocks ODK. One-carbon metabolism in cancer. Br J Cancer. 2017;116(12):1499-1504. doi:10.1038/bjc.2017.118 
852 56. Beydoun S, Choi HS, Dela-Cruz G, et al. An alternative food source for metabolism and longevity studies in Caenorhabditis elegans. Commun Biol. 2021;4(1). doi:10.1038/s42003-021-01764-4

57. Overmyer KA, Thonusin C, Qi NR, Burant CF, Evans CR. Impact of anesthesia and euthanasia on metabolomics of mammalian tissues: Studies in a C57BL/6J mouse model. PLoS One. 2015. doi:10.1371/journal.pone.0117232

58. Overmyer KA, Thonusin C, Qi NR, et al. Maximal oxidative capacity during exercise is associated with skeletal muscle fuel selection and dynamic changes in mitochondrial

59. Smith CA, Want EJ, O’Maille G, Abagyan R, Siuzdak G. XCMS: Processing mass spectrometry data for metabolite profiling using nonlinear peak alignment, matching, and identification. Anal Chem. 2006. doi:10.1021/ac051437y

60. Zhang Y, Chen D, Smith MA, Zhang B, Pan X. Selection of reliable reference genes in caenorhabditis elegans for analysis of nanotoxicity. PLoS One. 2012;7(3):31849. doi:10.1371/journal.pone.0031849

61. Motika MS, Zhang J, Zheng X, Riedler K, Cashman JR. Novel variants of the human flavin-containing monooxygenase 3 (FMO3) gene associated with trimethylaminuria. Mol Genet Metab. 2009;97(2):128-135. doi:10.1016/j.ymgme.2009.02.006 Untargeted Metabolomics. Anal Chem. 2019;91(3):2155-2162. 